\title{
ENTROPY OF BERGMAN MEASURES OF A TORIC KAEHLER MANIFOLD
}

\author{
PIERRE FLURIN AND STEVE ZELDITCH
}

\begin{abstract}
Associated to the Bergman kernels of a polarized toric Kähler manifold $(M, \omega, L, h)$ are sequences of measures $\left\{\mu_{k}^{z}\right\}_{k=1}^{\infty}$ parametrized by the points $z \in M$. We determine the asymptotics of the entropies $H\left(\mu_{k}^{z}\right)$ of these measures. The sequence $\mu_{k}^{z}$ in some ways resembles a sequence of convolution powers; we determine precisely when it actually is such a sequence. When $(M, \omega)$ is a Fano toric manifold with positive Ricci curvature, we show that there exists a unique point $z_{0}$ (up to the real torus action) for which $\mu_{k}^{z}$ has asymptotically maximal entropy. If the Kähler metric is Kähler -Einstein, we show that the image of $z_{0}$ under the moment map is the center of mass of the polytope. We also show that the Gaussian measure on the space $H^{0}\left(M, L^{k}\right)$ induced by the Kähler metric has maximal entropy at the balanced metric.
\end{abstract}

In [Z09], the second author introduced a sequence $\left\{\mu_{k}^{z}\right\}_{k=1}^{\infty}$ of probability measures on the convex lattice polytope $P \subset \mathbb{R}^{m}$ associated to a toric Kähler manifold $(M, \omega)$. The measures $\mu_{k}^{z}$ are supported on the dilated lattice points $P \cap \frac{1}{k} \mathbb{Z}^{m}$, and depend on a choice of Hermitian metric $h=e^{-\varphi}$ on the toric line bundle $L \rightarrow M$ with $\omega_{\varphi}:=i \partial \bar{\partial} \varphi=\omega$. They also depend on a point $z \in M$, or more precisely on its image $x=\mu(z)$ under the moment map

$$
\mu_{h}:=\mu: M \rightarrow P \subset \mathbb{R}^{m}
$$

associated to $h$. In the special case where $M=\mathbb{C P}^{1}$ and $\omega=\omega_{F S}$ is the Fubini-Study metric, the measures $\mu_{k}^{z}$ are the standard binomial measures indexed by $x \in[0,1]$ and coincide with the $k$ th convolution power $\mu_{x}^{* k}$ of the Bernoulli measure $\mu_{x}=x \delta_{1}+(1-x) \delta_{0}$ on $[0,1]$. More generally, for the Fubini-Study metric $h_{F S}$ on the kth power of the standard line bundle $\mathcal{O}(k) \rightarrow \mathbb{C P}^{m}$ in any dimension, the measures $\mu_{k}^{z}$ are the standard multi-nomial distributions, which are also a sequence of convolution powers. For general toric Kähler manifolds, the sequences $\left\{\mu_{k}^{z}\right\}_{k=1}^{\infty}$ is certainly not a sequence of convolution powers. Yet, many of the classical results on convolution powers are also valid for the sequence $\left\{\mu_{k}^{z}\right\}_{k=1}^{\infty}$ : In [SoZ12] they are shown to satisfy a law of large numbers and a large deviations principle; more recently, they were proved to satisfy a central limit theorem [ZZ18]. The purpose of this note is to given an asymptotic formula for the entropies of $\left\{d \mu_{k}^{z}\right\}_{k=1}^{\infty}$, extending the family of probabilistic results one step further. We further investigate the points $z$ and metrics $h$ for which the sequences have asymptotically maximal entropy. The proofs are non-probabilistic and are based on Bergman kernel asymptotics, and especially on the local CLT results in [ZZ18] and on the LDP in [SoZ12].

To state the result, we introduce some notation, referring to Section 1 and to [Z09, SoZ12, ZZ18] for much of the background. The moment map (1) associated to this data defines a torus bundle of the open orbit of $\left(\mathbb{C}^{*}\right)^{m}$ over the interior of the convex lattice polytope $P$. As reviewed in Section 1.2, there is a natural basis $\left\{s_{\alpha}\right\}_{\alpha \in k P}$ of the space $H^{0}\left(M, L^{k}\right)$ of holomorphic sections of the $k$-th power of $L$ by eigensections $s_{\alpha}$ of the $\mathbf{T}^{m}$ action. In a standard frame $e_{L}$ of $L$ over $M^{o}$, they correspond to monomials $z^{\alpha}$ on $\left(\mathbb{C}^{*}\right)^{m}$. The pointwise norms of $z^{\alpha}$ in the open orbit are given by $\left|z^{\alpha}\right|^{2} e^{-k \varphi(z)}$ where $h=e^{-\phi}$ in a standard frame. The toric Kähler potential $\phi$ on the open orbit is $\mathbf{T}^{m}$-invariant and may be viewed as a convex function on $\mathbb{R}^{m}$. Its Legendre transform $u$ is a convex function on $P$ known as the symplectic potential. For instance, the symplectic potential of the Fubini-Study metric is $u_{F S}(x)=x \log x+(1-x) \log (1-x)$ (see Section 1.3).

For $\alpha \in k P \cap \mathbb{Z}^{m}$, we define

$$
\mathcal{P}_{h^{k}}(\alpha, z):=\frac{\left|z^{\alpha}\right|^{2} e^{-k \varphi(z)}}{Q_{h^{k}}(\alpha)},
$$

Date: October 22, 2020.

Research partially supported by NSF grant DMS-1810747. and by the Stefan Bergman trust . 
where $Q_{h^{k}}(\alpha)$ is defined in (9). Further, we denote by $\Pi_{h^{k}}: L^{2}\left(M, L^{k}\right) \rightarrow H^{0}\left(M, L^{k}\right)$ the Szegö projector and by $\Pi_{h^{k}}(z)$ the associated density of states, i.e. the metric contraction of the diagonal of the kernel of $\Pi_{h^{k}}$; see Section 1.2. We now come to the main definition:

Definition 0.1. For any $z \in M^{o}$ and $k \in \mathbb{N}$, we define the probability measure on $P \subset \mathbb{R}^{m}$ by,

$$
\mu_{k}^{z}:=\frac{1}{\Pi_{h^{k}}(z, z)} \sum_{\alpha \in k P \cap \mathbb{Z}^{m}} \frac{\left|s_{\alpha}(z)\right|_{h^{k}}^{2}}{\left\|s_{\alpha}\right\|_{h^{k}}^{2}} \delta_{\frac{\alpha}{k}}=\frac{1}{\Pi_{h^{k}}(z, z)} \sum_{\alpha \in k P \cap \mathbb{Z}^{m}} \mathcal{P}_{h^{k}}(\alpha, z) \delta_{\frac{\alpha}{k}}
$$

Note that $\frac{1}{\Pi_{h^{k}}(z, z)} \sum_{\alpha \in k P \cap \mathbb{Z}^{m}} \mathcal{P}_{h^{k}}(\alpha, z)=1$. The measures are $\mathbf{T}^{m}$-invariant in $z$, and therefore define a family discrete measures on $P \cap \frac{1}{k} \mathbb{Z}^{m}$ parametrized by points $\mu_{h}(z) \in P$. Although it is not explicit in the notation, $\mu_{k}^{z}$ depends on the choice of Hermitian metric $h$ on $L$. For background on 'lattice probability measures' we refer to [GK].

REMARK 1. All of the techniques and result of this article can be extended to the case where $\mu(z) \in \partial P$, i.e. $z$ lies on the divisor at infinity of $M$. Indeed, the formulae derive from the large deviations principle of [SoZ12] and the convergence theorem for geodesics [SoZ07, SoZ10] and these results were proved for all z, including $z$ on the divisor at infinity. But since it is lengthier and more technical to work at the boundary, for the sake of brevity we assume $z \in M^{\circ}$ in this article.

0.1. Asymptotics of entropy of $\mu_{k}^{z}$. The (Shannon) entropy of a discrete probability measure with masses $\left\{p_{\alpha}\right\}$ is defined by (cf. [KS])

$$
H=-\sum_{\alpha} p_{\alpha} \ln p_{\alpha}
$$

Thus, the entropy of $\mu_{k}^{z}$ is

$$
H\left(\mu_{k}^{z}\right)=-\sum_{\alpha \in k P \cap \mathbb{Z}^{m}} \frac{\mathcal{P}_{h^{k}}(\alpha, z)}{\Pi_{h^{k}}(z)} \ln \frac{\mathcal{P}_{h^{k}}(\alpha, z)}{\Pi_{h^{k}}(z)} .
$$

The asymptotic entropy result is:

TheOREM 0.2. Let $h=e^{-\phi}$ be a toric Hermitian metric on $L \rightarrow M$ and let $\omega_{\varphi}=i \partial \bar{\partial} \phi$ be the corresponding Kähler metric. Then, as $k \rightarrow \infty$,

$$
H\left(\mu_{k}^{z}\right)=\frac{1}{2} \log \left(\operatorname{det}\left((2 \pi e k)\left(\left.i \partial \bar{\partial} \varphi\right|_{z}\right)\right)+o(1)\right.
$$

Note that the entropy depends only on the image $\mu_{h}(z)=x_{0}$ of $z$ under the moment map (1). Also, $\operatorname{det}(i \partial \bar{\partial} \varphi)$ is the density of the volume form $\omega_{\varphi}^{m}$ relative to Lebesgue measure on the open orbit. As in [Ab98] it is convenient to rewrite $\log \operatorname{det} i \partial \bar{\partial} \varphi$ in terms of the symplectic potential and its Hessian in action-angle variables, with action variables $x \in P$ and angle variables $\theta$ on $\mu_{h}^{-1}(x)$. We recall that the symplectic potential $u$ is the Legendre transform of the open orbit Kähler potential; we refer Section 1.3 and to [Ab98, Ab03] for background. Then set,

$$
L(x)=\frac{1}{2} \log \operatorname{det} \nabla^{2} u(x)=-\frac{1}{2} \log \operatorname{det} i \partial \bar{\partial} \varphi,
$$

and Theorem 0.2 may be reformulated as follows.

TheOREm 0.3. Let $h=e^{-\phi}$ be a toric Hermitian metric on $L \rightarrow M$ and let $u$ be the open orbit symplectic potential. Then, as $k \rightarrow \infty$,

$$
H\left(\mu_{k}^{z}\right)=\frac{1}{2} \log \left(\operatorname{det} \frac{(2 \pi e k)}{\left.\nabla^{2} u\right|_{\mu_{h}(z)}}\right)+o(1)=\frac{m}{2} \log (2 \pi e k)-L(x)+o(1) .
$$

Note that the entropy of uniform measure $\mu_{k P \cap \mathbb{Z}^{m}}$ on a set of $r$ element is $\log r$. The number $\#\left(k P \cap \mathbb{Z}^{m}\right)$ of such lattice points is $\simeq k^{m} \#\left(P \cap \mathbb{Z}^{m}\right)$, so that uniform measure on these lattice points has entropy $m \log k+\log \#\left(P \cap \mathbb{Z}^{m}\right) . \mu_{k}^{z}$ is not uniform, but rather is approximately a discretized Gaussian distribution centered at $\mu(z)$ and of width $k^{-\frac{1}{2}}$ (see Lemma 1.2 and Lemma 1.3 for the precise statements). A discretized Gaussian of width $k^{-\frac{1}{2}}$ and of height $k^{m}$ is concentrated in the ball $B\left(z, k^{-\frac{1}{2}}\right)$ and is similar to uniform measure on that ball of the same height. This approximation accurately predicts the leading order term $\log k^{m / 2}$. 
REMARK 2. One may expect analogous results for non-compact infinite volume toric Kähler manifolds, such as $\mathbb{C}^{m}$ with the Bargmann-Fock space of analytic functions. The techniques of [F12] apply in that setting. However, the large deviations results have not been established in such cases, and we confine the article to compact Kähler manifolds.

Theorem 0.3 specializes to known asymptotics of entropies of multinomial distributions when $(M, \omega)$ is complex projective space with Fubini-Study metric. In dimension $m=1$, the binomial distributions are convolution powers $\mu_{k}^{p}=\left(\mu_{p}\right)^{* k}$ of the Bernoulli measure $\mu_{p}$ defined by $\mu_{p}(\{1\})=p, \mu_{p}(\{0\})=1-p$. In this case, the entropy asymptotics can be obtained from local central limit theorems and Stirling's formula, and according to [JSz99, Theorem 2] and to [K98]), $H\left(\mu_{k}^{z}\right)$ has a complete asymptotic expansion in powers of $k^{-1}$ whose coefficients involve the Bernoulli numbers. The entropy of $\mu_{p}$ is $p \log p+(1-p) \log (1-p)=u_{F S}(p)$, the Fubini-Study symplectic potential (see Section 1.3 and [Ab98] for background). Thus, $p(1-p)=\left(u_{F}^{\prime \prime}(p)\right)^{-1}$. The parameter $p \in[0,1]$ is the image of the parameter $z \in \mathbb{C P}^{1}$ under the Fubini-Study moment map. The $k$ th convolution power $\mu_{k}^{p}$ is the binomial measure, for which $p_{k, \ell}=\left(\begin{array}{c}k \\ \ell\end{array}\right) p^{\ell}(1-p)^{k-\ell}$. Its Shannon entropy has the asymptotics (see [JSz99, Corollary 1]),

$$
H\left(\mu_{k}^{p}\right)=\frac{1}{2} \log k+\frac{1}{2}\left(1+\log (2 \pi p(1-p))+O\left(k^{-\frac{1}{2}}+\epsilon\right) .\right.
$$

To compare with Theorem 0.3, we note that in the Fubini-Study case, $u_{F S}^{\prime}(x)=\log \frac{x}{1-x}, u_{F S}^{\prime \prime}(x)=\frac{1}{x(1-x)}$, $\log \left(u_{F S}^{\prime \prime}(x)\right)^{-1}=\log x(1-x)$.

Now consider multinomial distributions, which correspond to the toric Kähler manifold $M=\mathbb{C P}^{m}$ with the Fubini-Study metric $h_{F S}$ on $L=\mathcal{O}(1)$. The parameters $\vec{p}$ corresponds to a point in $\Delta_{m}=\left\{\vec{p} \in \mathbb{R}_{+}^{m+1}\right.$ : $\left.\sum_{j=1}^{m+1} p_{j}=1\right\}$, which is polytope associated to $\mathbb{C P}^{m}$. Given $k \in \mathbb{Z}_{\geq 1}$, let $\vec{\alpha} \in \mathbb{Z}_{+}^{m+1}$ and let $k=|\vec{\alpha}|$. If $\vec{x} \in \mathbb{R}^{m+1}$ let $\vec{x}^{\vec{\alpha}}=\prod_{j=1}^{m+1} x_{1}^{\alpha_{1}} \cdots x_{m+1}^{\alpha_{m+1}}$. A random vector $\vec{X}=\left(X_{1}, \ldots, X_{m+1}\right)$ has the multinomial distributions with parameters $k$ and $\vec{p}$ if $\operatorname{Prob}[\tilde{\mathrm{X}}=\tilde{\alpha}]=\left(\begin{array}{c}\mathrm{k} \\ \tilde{\alpha}\end{array}\right) \tilde{\mathrm{p}}^{\tilde{\alpha}}$ where $\left(\begin{array}{l}k \\ \vec{\alpha}\end{array}\right)=\frac{k !}{\tilde{\alpha} !}$.

It is proved in [CG12, Theorem 1] and [Mat78] that the multinomial distributions with parameters $k$ and $\vec{p}=\left(p_{1}, \ldots, p_{m+1}\right)$ has the asymptotic form,

$$
H\left(\mu_{p}^{* k}\right)=\frac{1}{2} \log \left((2 \pi k e)^{m} p_{1} \cdots p_{m+1}\right)+\frac{1}{12 k}\left(3(m+1)-2-\sum_{j=1}^{m+1} \frac{1}{p_{j}}\right)+O\left(\frac{1}{k^{2}}\right) .
$$

REMARK 3. Since $\sum_{j=1}^{m+1} p_{j}=1$, there are only $m$ independent $p_{j}$. In the formula of Theorem 0.2, the parameter $m+1$ in the multinomial case corresponds to $\mathbb{C P}^{m}$, so the coefficients of log $k$ agree.

Aside from asymptotic entropies of multinomial distributions, there exist few general results on asymptotic entropies of convolution powers $\mu^{* k}$. Asymptotics of entropies to several orders for certain classes of discrete distributions as $k \rightarrow \infty$ were obtained in [K98, JSz99]. In the case of sums of i.i.d. real-valued random variables, i.e. convolution powers of probability measures on $\mathbb{R}$, Dyachkov proved in [D96, Theorem 2] that

$$
H\left(\mu^{* k}\right) \simeq \frac{1}{2}(\log k)+\frac{1}{2} \log \left(2 \pi e \sigma^{2}\right)+o(1) .
$$

In view of the resemblence of the entropy asymptotics of the toric Kähler probability measures $\mu_{k}^{z}$ to convolution powers, it is natural to characteristic the toric Hermitian line bundles $(L, h) \rightarrow(M, \omega)$ for which $\mu_{k}^{z}$ is a sequence of convolution powers.

THEOREM 0.4. The sequence $\left\{\mu_{k}^{z}\right\}_{k=1}^{\infty}$ is a sequence of convolution powers for all $z$ if and only if $\mathrm{Hilb}_{\mathrm{k}}(\mathrm{h})$ is balanced for all $k$, i.e. the density of states $\Pi_{h^{k}}(z)=D_{k}$ is constant for all $k$. Hence, $\omega$ is a Kähler metric of constant scalar curvature;

To prove Theorem 0.4 we first prove a result about balanced metrics on any Kähler manifold which seems of independent interest.

Proposition 0.5. For any Kähler manifold $(M, \omega, J)$, the following are equivalent:

(1) $\operatorname{Hilb}_{\mathrm{k}}(\mathrm{h})$ is balanced for all $k$, i.e. the density of states $\Pi_{h^{k}}(z)=C_{k}$ is constant for all $k$. 
(2) $\Pi_{h^{k}}(z, w)=A_{k}\left[\Pi_{h^{1}}(z, w)\right]^{k}$, where

$$
A_{k}=\frac{\operatorname{dim} H^{0}\left(M, L^{k}\right)}{(2 \pi)^{m} \operatorname{Vol}(P)}\left(\frac{(2 \pi)^{m} \operatorname{Vol}(P)}{\operatorname{dim} H^{0}(M, L)}\right)^{k} .
$$

In the case of a toric Kähler manifold,

$$
A_{k}=\left(\frac{\#\left\{\alpha \in k \bar{P} \cap \mathbb{Z}^{m}\right\}}{(2 \pi)^{m} \operatorname{Vol}(P)}\right)\left(\frac{(2 \pi)^{m} \operatorname{Vol}(P)}{\#\left\{\alpha \in \bar{P} \cap \mathbb{Z}^{m}\right\}}\right)^{k} .
$$

We refer to [D02] for background and results on balanced and constant scalar curvature metrics on toric Kähler manifolds.

0.2 . Ricci curvature and measures of maximal entropy. The entropy $H(\mu)$ of a discrete probability measure $\mu$ is a measure of the degree to which $\mu$ is uniform. The larger the entropy, the more uniform the measure, so that the measure of maximal entropy in a given family of probability measures is the most uniform measure. This measure of maximal entropy is often considered the most important. Hence it is natural to ask for which $z$ does $\mu_{k}^{z}$ have maximal entropy in the family $\mu_{k}^{z}$, at least asymptotically as $k \rightarrow \infty$. For instance, in the case of binomial measures $\mu_{p}^{* k}, p=\frac{1}{2}$.

Locating the point $\mu(z)=x$ where $\mu_{k}^{z}$ has asymptotically maximal entropy is related to the Ricci curvature of $(M, \omega)$. We recall that the Ricci curvature of the Kähler metric $\omega_{\varphi}$ is given by $\operatorname{Ric}(\omega)=-\mathrm{i} \partial \bar{\partial} \log \operatorname{det}\left(\mathrm{g}_{\mathrm{ij}}\right)$, i.e. $\operatorname{Ric}_{\mathrm{k} \ell}=-\frac{\partial^{2}}{\partial \mathrm{z}_{\mathrm{k}} \partial \overline{\mathrm{z}}_{\ell}}\left(\log \operatorname{det} \mathrm{g}_{\mathrm{i} \mathrm{j}}\right)$ where $\omega=\frac{i}{2} g_{i \bar{j}} d z^{i} \wedge d \bar{z}^{j}$. In [Ab98] it is shown that in the toric case,

$$
\text { Ric }=-\frac{1}{2} \mathrm{dd}^{\mathrm{c}} \log \operatorname{det} \mathrm{H}=-\frac{1}{2} \sum_{\mathrm{i}, \mathrm{j}, \mathrm{k}}^{\mathrm{m}} \mathrm{H}_{\mathrm{ij}, \mathrm{jk}} \mathrm{dx}_{\mathrm{k}} \wedge \mathrm{d} \theta_{\mathrm{i}},
$$

Thus, the Ricci potential is the function $-L(x)(5)$.

Due to the inverse relation of $i \partial \bar{\partial} \phi$ and $\nabla^{2} u$, points where the Ricci potential is maximal are points where (5) is minimal. In the simplest case of the Fubini-Study symplectic potential on $\mathbb{C P}^{1}$, in a standard gauge the symplectic potential satisfies, $\log u_{F S}^{\prime \prime}(x)=-\log x(1-x)$, and $\frac{d^{2}}{d x^{2}} \log u_{F S}^{\prime \prime}(x)=x^{-2}+(1-x)^{-2}$. The unique minimum point of $\log u_{F S}^{\prime \prime}$ occurs at $x=\frac{1}{2}$. In the case of multinomial distributions and Fubini-Study potentials in higher dimensions, the maximum occurs at the center of mass of the simplex. These are model cases of toric Fano Kähler -Einstein manifolds. It turns out that related statements are true for compact toric Kähler manifolds with positive Ricci curvature. We recall that $\operatorname{Ric}(\omega)$ represents the first Chern class $c_{1}(M)$ and Ric $>0$ implies that $(M, \omega)$ is a toric Fano manifold. That is, if $\operatorname{Ric}(\omega)>0$, then $\omega$ is a positively curved metric on the anti-canonical bundle $-K_{X}$, hence $-K_{X}$ is ample. A toric Fano manifold has a distinguished center, namely the center of mass of polytope. We refer to [D08] for background and results on toric Fano Kähler manifolds and their preferred centers.

Theorem 0.6. For fixed $(L, h, M, \omega)$, the points $x=\mu(z)$ for which the measures $\mu_{k}^{z}$ have asymptotically maximal entropy as $k \rightarrow \infty$ occur at the minimum points of $L(x)(5)$. If $(M, \omega)$ is Fano and $\operatorname{Ric}(\omega)$ is positive, then there is a unique minimum. In the Kähler-Einstein Fano case, where $\operatorname{Ric}(\omega)=a \omega$, the point of maximal entropy is the center of mass of $P$ (which equals 0 if $P$ is put in the form of [M87].)

For instance, in the case of Fubini-Study metrics on $\mathbb{C P}^{m}$, the open orbit Kähler potential is $\log (1+$ $\left.|w|^{2}\right), w \in \mathbb{C}^{m}$, and $-\log \operatorname{det} \nabla^{2} \varphi(\rho)=(m+1) \varphi(\rho)-\rho$. The unique point of maximal entropy is given by $\frac{e^{\rho}}{1+e^{\rho}}=\frac{1}{m}(1, \ldots, 1)$. In the gauge of Mabuchi [M87], where the polytope is translated by $-\frac{1}{m}(1, \ldots, 1)$, the unique point is 0 (see Section 1.4 for background on gauges).

REMARK 4. The Mabuchi functional $\mathcal{M}(\omega)$ on Kähler metrics involves the relative entropy of $\omega_{\phi}^{m}$ and of a background volume form. As shown in [D02, Proposition 3.2.8], it is given on a toric Kähler manifold by $\mathcal{M}(\omega)=(2 \pi)^{n} \mathcal{F}_{a}(u)$ where,

$$
\mathcal{F}_{a}(u)=\int_{P} L(x) d x+\int_{\partial P} u d \sigma-a \int_{P} u d x,
$$

where $a=\frac{\operatorname{Vol}(\partial \mathrm{P}, \mathrm{d} \sigma)}{\operatorname{Vol}(\mathrm{P})}$ where $d \sigma$ is Euclidean surface measure. 
0.3. Differential entropy of the Gaussian measure $\gamma_{h^{k}}$. There is a second (and much simpler) problem regarding entropies of probability measures on a toric Kähler manifold, or indeed on any polarized Kähler manifold. Associated to any Hermitian metric $h$ on $L$ is a sequence $\left\{\text { Hilb }_{\mathrm{k}}(\mathrm{h})\right\}_{\mathrm{k}=1}^{\infty}$ of Hermitian inner products on $H^{0}\left(M, L^{k}\right)$. In turn the inner product induces a Gaussian measure $\gamma_{h_{k}}$ on $H^{0}\left(M, L^{k}\right)$. If we fix a background metric $h_{0}$, or corresponding inner product $G_{0}$, then the inner product Hilb b $_{\mathrm{k}}$ is represented by a positive Hermitian matrix $P$ and the Gaussian measure $\gamma_{k}^{h}$ is represented by $\operatorname{det} P e^{-\left\langle P^{-1} X, X\right\rangle}$ on $\mathbb{C}^{N_{k}}$ where $N_{k}=\operatorname{dim}_{\mathbb{C}} H^{0}\left(M, L^{k}\right)$,

When a probability measure $\mu$ on $\mathbb{R}^{n}$ has a density $f$ relative to Lebesgue measure $d x$, its differential entropy is defined by

$$
H(f d x)=-\int_{\mathbb{R}^{n}} f(x) \log f(x) d x .
$$

It is well-known that if $f(x)=N(\mu, \sigma)=\frac{1}{\sqrt{2 \pi} \sigma} \exp \left(-\frac{(x-\mu)^{2}}{2 \sigma^{2}}\right)$ is a Gaussian, then,

$$
h(f d x)=\ln (\sigma \sqrt{2 \pi e}) .
$$

We now calculate the differential entropy of the Gaussian measures $\gamma_{k}^{h}$.

Proposition 0.7. Let $(L, h, M, \omega)$ be any polarized Kähler manifold, and let $\gamma_{k}^{h}$ be the associated Gaussian measure on $H^{0}\left(M, L^{k}\right)$. Then $H\left(\gamma_{k}^{h}\right)=-\log \operatorname{det} H_{i l b}(\mathrm{~h})$. The Hermitian metric h for which $H\left(\gamma_{k}^{h}\right)$ has maximal entropy is the balanced metric.

0.4. Further problems on the sequence of toric measures. Although entropy has a natural interpretation for a single probability measure (its degree of uniformity), it plays a more essential role in the dynamics of Markov chains (the Shannon-Breiman-MacMillan theorem; see [KS]).

A well-known Markov chain is the so-called Wright-Fisher Markov chain: Let $X_{n}(n \geq 0)$ be the Markov chain with state space $\{0, \ldots, N\}$ and with transition probabilities,

$$
\left.p_{i j}:=P\left(X_{n+1}\right)=j \mid X_{n}=i\right)=\left(\begin{array}{c}
N \\
j
\end{array}\right)\left(\frac{i}{N}\right)^{j}\left(1-\frac{i}{N}\right)^{N-j}, \quad(i, j=0, \ldots, N) .
$$

A more general Wright-Fisher Markov chain is to define

$$
p_{i j}=\left(\begin{array}{c}
N \\
j
\end{array}\right) \psi_{i}^{j}\left(1-\psi_{i}\right)^{N-j},
$$

where $\psi_{i}$ are other weights of the lattice points. There is a straightforward generalization to toric Kähler manifolds by defining the right stochastic matrix

$$
P_{\alpha \beta}^{(N)}=\frac{\left|s_{\alpha}(\beta)\right|_{h^{N}}^{2}}{Q_{h^{N}}(\alpha) \Pi_{h^{N}}(\beta, \beta)} .
$$

In fact, we could take any orthonormal basis $\left\{s_{N, j}\right\}$ and any points $\left\{z_{N, k}\right\}_{k=1}^{d_{N}}$ and form the Markov chain with

$$
P_{j, k}^{(N)}:=\frac{\left|s_{N, j}\left(z_{N, k}\right)\right|_{h^{N}}^{2}}{\Pi_{h^{N}}\left(z_{N, k}, z_{N, k}\right)} .
$$

It might be of interest to determine the asymptotic entropy of this Markov chain, which is closely related to the measures $\mu_{k}^{z}$.

REMARK 5. The article $[\mathrm{DK}]$ also considers entropy in the context of Bergman kernels, but does not seem to overlap this article. It is devoted to the simpler question of when the density of states $\Pi_{h^{k}}(z)$ has maximal entropy (it is evidently the balanced metric) and its applications to black hole physics.

0.5. Acknowledgements. Thanks to Peng Zhou for many helpful conversations, particularly about gauge freedom for toric Kähler manifolds. Thanks also to the referee for pointing out some errors and omissions in an earlier version, which led the authors to add Theorem 0.6. 


\section{BACKGROUND ON TORIC VARIETIES}

We employ the same notation and terminology as in [SoZ12, ZZ18]. We recall that a toric Kähler manifold is a Kähler manifold $(M, J, \omega)$ on which the complex torus $\left(\mathbb{C}^{*}\right)^{m}$ acts holomorphically with an open orbit $M^{o}$. We choose a basepoint $z_{0}$ on the orbit open and identify $M^{o} \equiv\left(\mathbb{C}^{*}\right)^{m}\left\{z_{0}\right\}$. The underlying real torus is denoted $\mathbf{T}^{m}$ so that $\left(\mathbb{C}^{*}\right)^{m}=\mathbf{T}^{m} \times \mathbb{R}_{+}^{m}$, which we write in coordinates as $z=e^{\rho / 2+i \theta}$ in a multi-index notation. Thus, $|z|^{2}=e^{\rho}$. We often express the Kähler potential in $\rho$ coordinates.

We assume that $M$ is a smooth projective toric Kähler manifold, hence that $P$ is a Delzant polytope, i.e. that $P$ is defined by a set of linear inequalities

$$
\ell_{r}(x):=\left\langle x, v_{r}\right\rangle-\alpha_{r} \geq 0, r=1, \ldots, d,
$$

where $v_{r}$ is a primitive element of the lattice and inward-pointing normal to the $r$-th $(n-1)$-dimensional face of $P$. We denote by $P^{o}$ the interior of $P$ and by $\partial P$ its boundary; $P=P^{o} \cup \partial P$.

1.1. Toric line bundles and their powers. We consider powers $L^{k} \rightarrow M$ of an ample toric line bundle $L \rightarrow M$ with $k \in \mathbb{Z}$. A model case is that of powers $\mathcal{O}(k) \rightarrow \mathbb{C P}^{m}$ of the dual line bundle $\mathcal{O}(1) \rightarrow \mathbb{C P}^{m}$ of the hyperplane line bundle $\mathcal{O}(-1) \rightarrow \mathbb{C P}^{m}$. Positive powers $\mathcal{O}(k)$ are ample line bundles and the holomorphic sections correspond to monomials $z^{\alpha}$ on $\mathbb{C}^{m+1}$ with $|\alpha|=k$. Negative powers have no holomorphic sections.

The canonical line bundle $\mathcal{K}$ is the top exterior power of the holomorphic cotangent bundle; its sections are smooth $(m, 0)$ volume forms. Theorem 0.6 concerns Fano toric Kähler manifolds, namely manifolds with ample anti-canonical line bundle (hence, negative canonical line bundle). A model example is $\mathbb{C P}^{m}$, for which $\mathcal{K}=\mathcal{O}(-(m+1))$.

We need to linearize (or quantize) the torus action so that it acts on $H^{0}\left(M, L^{k}\right)$. It is sufficent to lift the action to $L^{*}$. The lifting procedure is described in [ZZ19, Lemma 1.1] for single Hamiltonians, and essentially the same procedure works to define lifts of the commuting Hamiltonians of a torus action. We equip $L$ with a toric Hermitian metric $h$ whose curvature $(1,1)$-form $\omega$. The Hermitian metric $h$ on $L$ induces a Chern connection on the $S^{1}$ bundle $X_{h}=\partial D_{h}^{*} \rightarrow M$ where $D_{h}^{*} \subset L^{*}$ is the unit co-disk bundle with respect to $h$. We then lift the Hamilton vector fields $\xi_{H_{j}}$ generating the torus action on $M$ to contact vector fields $\hat{\xi}_{H_{j}}=\xi_{H_{j}}^{h}-2 \pi H_{j} R$ on $X_{h}$ where $\hat{\xi}_{H}^{h}$ denotes the horizontal lift of $\xi_{H}$ and where $R$ is the Reeb vector field generating rotations in the fibers of $X_{h} \rightarrow M$. The vertical and horizontal parts commute, and if $\xi_{H_{j}}$ commute then their horizontal lifts commute. We may choose generators so each $\xi_{H_{j}}$ generates a $2 \pi$-periodic flow (such Hamiltonians are known as action variables). It is verified in [ZZ19, Lemma 2.6] that $\hat{\xi}_{H_{j}}$ also generates a $2 \pi$-periodic flow. Together with $R$, one has a $\mathbb{T}^{m+1}$ action on $X_{h}$. The Hamiltonians $H_{j}$ are not uniquely defined because one may add a constant $c_{j}$ to each without changing $\xi_{H_{j}}$. However, in order that the lifts $\hat{\xi}_{H_{j}}$ generate periodic flows, it is only possible to add a lattice point $\vec{k} \in \mathbb{Z}^{m}$ to the vector $\left(H_{1}, \ldots, H_{m}\right)$ of Hamiltonians. Thus, the possible lifts form a $\mathbb{Z}^{m}$-family.

For each choice of lift and each power $L^{k}$ of the ample toric line bundle, there exists a unique (up to scalars) torus-invariant section, whose restriction to the open orbit we denote by $e_{L^{k}}$. See [Fu] or [GS82, Section 5] (which treats general compact Lie groups). In the case of $\mathcal{O}(k) \rightarrow \mathbb{C P}^{m}$ it corresponds to the lattice point $\alpha=0$. For $k=m+1$, the invariant section may be viewed as the multivector $\left(z_{1} \frac{\partial}{\partial z_{1}}\right) \wedge \cdots \wedge\left(z_{m} \frac{\partial}{\partial z_{m}}\right)$ dual to the meromorphic invariant volume form $\frac{d z_{1}}{z_{1}} \wedge \cdots \wedge \frac{d z_{m}}{z_{m}}$, which has an order 1 pole at each boundary divisor.

A natural basis of the space of holomorphic sections $H^{0}\left(M, L^{k}\right)$ associated to the $k$ th power of an ample toric holomorphic line bundle $L \rightarrow M$ is the basis of equivariant sections, i.e. holomorphic eigensections of the linearized torus action. They are defined in the open orbit by the monomials $z^{\alpha}$ where $\alpha$ is a lattice point in the $k$ th dilate of the polytope, $\alpha \in k P \cap \mathbb{Z}^{m}$. To be more precise, on the open orbit $s_{\alpha}(z)=z^{\alpha} e_{L^{k}}$. Let $I_{k} \subset \mathbb{Z}^{m}$ be the subset consisting of the weights in $H^{0}\left(M, L^{k}\right)$ under the action of $\left(\mathbb{C}^{*}\right)^{m}$, and let $P_{k}$ be the convex hull of $I_{k}$. Then $P_{k}=k P^{\prime}$ for a fixed convex polytope $P^{\prime}$. We denote the dimension of $H^{0}\left(M, L^{k}\right)$ by $N_{k}$. For background, see [Fu].

1.2. Inner products and norms of monomials. We equip $L$ with a toric Hermitian metric $h$ whose curvature $(1,1)$-form may be expressed in terms of a local holomorphic frame $e_{L}$ by $\omega=i \partial \bar{\partial} \log \left\|e_{L}\right\|_{h}^{2}$. Any 
hermitian metric $h$ on $L$ induces inner products $\operatorname{Hilb}_{k}(h)$ on $H^{0}\left(M, L^{k}\right)$, defined by

$$
\left\langle s_{1}, s_{2}\right\rangle_{\operatorname{Hilb}_{k}(h)}=\int_{M}\left(s_{1}(z), s_{2}(z)\right)_{h^{k}} \frac{\omega_{h}^{m}}{m !} .
$$

The equivariant sections (monomials) are orthogonal with respect to any such toric inner product. We often express the norm in terms of a local Kähler potential, $\left\|e_{L}\right\|_{h}^{2}=e^{-\varphi}$, so that $\left|s_{\alpha}(z)\right|_{h^{k}}^{2}=\left|z^{\alpha}\right|^{2} e^{-k \varphi(z)}$ for $s_{\alpha} \in H^{0}\left(M, L^{k}\right)$. The $L^{2}$ norm-square of $s_{\alpha}$ with respect to the natural inner product $\operatorname{Hilb}_{k}(h)$ induced by the Hermitian metric on $H^{0}\left(M, L^{k}\right)$ is given by,

$$
Q_{h^{k}}(\alpha)=\left\|s_{\alpha}\right\|_{h^{k}}^{2}=\int_{\mathbb{C}^{m}}\left|z^{\alpha}\right|^{2} e^{-k \varphi(z)} d V_{\varphi}(z)
$$

Here, $d V_{\varphi}=(i \partial \bar{\partial} \varphi)^{m} / m !$.

1.3. Kähler potential, moment map and sympletic potential. An open-orbit Kähler potential is a real-valued torus invariant function $\varphi$ such that $i \partial \bar{\partial} \varphi=\omega$ on the open orbit. This potential is only defined up to an additive pluri-harmonic torus invariant function (i.e. an affine function). Recall that the $\log$ coordinates $(\rho, \theta)$ on $M^{o} \cong\left(\mathbb{C}^{*}\right)^{m}$ are defined by setting $z_{i}=e^{\rho_{i} / 2+\sqrt{-1} \theta_{i}}$. Since the Kahler potential $\varphi$ is $\mathbf{T}^{m}$-invariant, $\varphi(z)$ only depends on the $\rho$ variables, hence we may write it as $\varphi(\rho)\left(e^{\rho}=|z|^{2}\right)$. The associated moment map $\mu_{h}$ is defined as the gradient of the Kähler potential,

$$
\mu_{h}: \mathbb{R}_{\rho}^{m} \rightarrow P \subset \mathbb{R}_{x}^{m}, \quad \rho \mapsto \nabla \phi(\rho) .
$$

The polytope $P$ is the image of the moment map. The moment map $\mu_{h}: M \rightarrow \mathbb{R}^{m}$ is only well-defined up to an additive constant vector; hence $P$ is only defined up to translation without further normalization. As this indicates, there are a number of implicit choices in the definition of the Kähler potential and moment map.

Given the norming constants (9), a standard definition of the open-orbit Kähler potential is,

$$
\varphi(\rho):=\log \left(\sum_{\alpha \in P \cap \mathbb{Z}^{m}} \frac{e^{\langle\alpha, \rho\rangle}}{Q_{h}(\alpha)}\right) .
$$

The sum is essentially the density of states (the value of the Bergman kernel on the diagonal) (see Section 1.6). More precisely, it is the modulus square of the coefficient of the Bergman kernel relative to the invariant frame, i.e. the ratio of the density of states and the norm square $\left|e_{L}\right|^{2}$ of the invariant section. These definitions depend on the choice of linearization of the torus action. If the invariant section corresponds to the lattice point $\alpha_{0}$, then the exponent in $e^{\langle\alpha, \rho\rangle}$ in (10) is translated to $e^{\left\langle\alpha-\alpha_{0}, \rho\right\rangle}$. $\mathbb{R}^{m}:$

We now consider the symplectic potential $u_{0}$ associated to $\phi_{P^{\circ}}$, defined as the Legendre transform of $\varphi$ on

$$
u_{\varphi}(x)=\varphi^{*}(x)=\mathcal{L} \varphi(x):=\sup _{\rho \in \mathbb{R}^{m}}\left(\langle x, \rho\rangle-\varphi\left(e^{\rho / 2+i \theta}\right)\right) .
$$

It is a function on $P$, or in invariant terms it is a function on $\operatorname{Lie}\left(\mathbf{T}^{m}\right)^{*} \simeq \operatorname{Lie}\left(\mathbb{R}_{+}^{m}\right)^{*}$. In general, the Legendre transform of a function on a vector space $V$ is a function on the dual space $V^{*}$.

Thus,

$$
u_{\varphi}(x)=\left\langle x, \rho_{x}\right\rangle-\varphi\left(\rho_{x}\right), \quad e^{\rho_{x} / 2}=\mu_{\varphi}^{-1}(x) \Longleftrightarrow \rho_{x}=2 \log \mu_{\varphi}^{-1}(x)
$$

on $P$. The gradient $\nabla_{x} u_{\varphi}$ is an inverse to $\mu_{\omega_{\varphi}}$ on $M_{\mathbb{R}}$ on the open orbit, or equivalently on $P$, in the sense that $\nabla u_{\varphi}\left(\mu_{\omega_{\varphi}}(z)\right)=z$ as long as $\mu_{\omega_{\varphi}}(z) \notin \partial P$.

The symplectic potential has canonical logarithmic singularities on $\partial P$. According to [Gu94] and to [D02] ( Proposition 3.1.7),

$$
u_{0}(x)=\sum_{k} \ell_{k}(x) \log \ell_{k}(x)+f_{0}
$$

where $f_{0} \in C^{\infty}(\bar{P})$. The Guillemin canonical metric is the special case where $f_{0}=0$. 
PIERRE FLURIN AND STEVE ZELDITCH

1.4. Gauges and examples. As mentioned above, Kähler potentials and the corresponding symplectic potentials are not unique, and we refer to a choice of one potential as a choice of gauge. The gauge symmetries of the pairs $(\varphi, u)$ are as follows. We assume that the arguments $\rho$ of $\varphi$ and $x$ of $u$ are related by $x=\mu_{h}\left(e^{\rho / 2}\right)$.

- $\varphi \rightarrow \varphi+c, u \rightarrow u-c$ for any $c \in \mathbb{R}$;

- $\varphi(\rho) \rightarrow \varphi(\rho)+\vec{b} \cdot \rho, x \rightarrow x+\vec{b}=\nabla(\varphi(\rho)+\vec{b} \cdot \rho) \vec{b} \in \mathbb{R}^{m}$;

- $u(x) \rightarrow u(x)+\vec{k} \cdot x, \rho \rightarrow \rho+\vec{k}$.

- If we only choose Kähler potentials (10) corresponding to Bergman kernels and torus-invariant sections, then $\vec{b} \in \mathbb{Z}^{m}$.

Let us illustrate the definitions and ambiguities with the Kähler potential, moment map and symplectic potential for the Fubini-Study metric of $\mathbb{C P}^{m}$. In the case of $\mathcal{O}(1) \rightarrow \mathbb{C P}^{1}$, a standard choice for the FubiniStudy Kähler potential is $\varphi(z)=\log \left(1+|z|^{2}\right)=\log \left(1+e^{\rho}\right)=\varphi\left(e^{\rho}\right)=\varphi(\rho)$ (with a little abuse of notation) and the moment map is $\mu_{F S}(\rho)=\frac{e^{\rho}}{1+e^{\rho}}$. As in the introduction, the polytope is $[0,1]$ and the correspondinng symplectic potential is $u_{F S}(x)=x \log x+(1-x) \log (1-x)$.

However, other gauge choices are possible. If we allow all Chern classes and intervals, a second choice of Kähler potential is $\varphi_{r}(\rho)=r^{2} \log \cosh \rho$. The corresonding symplectic potential is,

$$
u_{r}(x)=\frac{1}{2}\left(\left(r^{2}+x\right) \log \left(r^{2}+x\right)+\left(r^{2}-x\right) \log \left(r^{2}-x\right)\right),
$$

where the parameter $r$ determines the radius of the corresponding round $S^{2}$, and corresponding polytope is $\left[-r^{2}, r^{2}\right]$. The radius parametrizes the cohomology class of the Kähler form. which is a translate of the centered polytope $\left[-\frac{1}{2}, \frac{1}{2}\right]$ with $r^{2}=\frac{1}{2}$.

As a higher dimensional example, consider the canonical bundle $\mathcal{O}(-(m+1)) \rightarrow \mathbb{C P}^{m}$ with the FubiniStudy metric. The image of the moment map for this potential is $(m+1)$ times the standard unit simplex in $\mathbb{R}^{m}$. The volume form is given by $\omega_{F S}^{m}=\left(\frac{i}{2}\right)^{m} \frac{\prod_{i=1}^{m} d z_{i} \wedge d \bar{z}_{i}}{\left(1+|z|^{2}\right)^{m+1}}$ and $-\log \left(1+|z|^{2}\right)^{m+1}$ is a Kähler potential for the Ricci form. On the other hand, one may express the volume form in terms of the invariant $(m, 0)$ form $\prod_{i=1}^{m} \frac{d z_{i}}{z_{i}}$. That changes the Kähler potential by $-\sum_{j} \log \left|z_{j}\right|$ and translates the simplex by $-(1,1, \cdots, 1)$ so that it is centered at 0 .

1.5. Fano toric Kähler manifolds. Theorem 0.6 pertains to Fano toric Kähler manifolds, namely those with positive anti-canonical bundle. The polytope of a Fano Kähler manifold has a preferred center $x_{0} \in P$ such that $\ell_{j}\left(x_{0}\right)=1$ for all $j$. As explained in [D08, Section 3.1], this follows because the wedge product of the vector fields generating the torus action is a meromorphic m-form on $M$ with a simple pole along each of the divisors corresponding to the faces. Its inverse is a section of the anti-canonical bundle. The centre $x_{0}$ is also the centre of mass of $\partial P$ with its induced surface measure. The center is 0 if and only if the metric is Kähler -Einstein [M87, WZ04]; equivalently, vanishing of the Futaki invariant is equivalent to the fact that the preferred center is the center of mass.

1.6. Szegö (or, Bergman) kernels. The Szegö (or Bergman) kernels of a positive Hermitian line bundle $(L, h) \rightarrow(M, \omega)$ are the kernels of the orthogonal projections $\Pi_{h^{k}}: L^{2}\left(M, L^{k}\right) \rightarrow H^{0}\left(M, L^{k}\right)$ onto the spaces of holomorphic sections with respect to the inner product $\operatorname{Hilb}_{k}(h)$,

$$
\Pi_{h^{k}} s(z)=\int_{M} \Pi_{h^{k}}(z, w) \cdot s(w) \frac{\omega_{h}^{m}}{m !},
$$

where the denotes the $h$-hermitian inner product at $w$. In terms of a local frame $e$ for $L \rightarrow M$ over an open set $U \subset M$, we may write sections as $s=f e$. If $\left\{s_{j}^{k}=f_{j} e_{L}^{\otimes k}: j=1, \ldots, N_{k}\right\}$ is an orthonormal basis for $H^{0}\left(M, L^{k}\right)$, then the Szegö kernel can be written in the form

$$
\Pi_{h^{k}}(z, w):=F_{h^{k}}(z, w) e_{L}^{\otimes k}(z) \otimes \overline{e_{L}^{\otimes k}(w)},
$$

where

$$
F_{h^{k}}(z, w)=\sum_{j=1}^{N_{k}} f_{j}(z) \overline{f_{j}(w)}, N_{k}=\operatorname{dim} H^{0}\left(M, L^{k}\right)
$$


We also introduce the local kernel $B_{k}(z, w)$, defined with respect to the unitary frame:

$$
\Pi_{h^{k}}(z, w)=B_{k}(z, w) \cdot \frac{e_{L}^{k}(z)}{\left\|e_{L}^{k}(z)\right\|_{h}} \otimes \frac{\overline{e_{L}^{k}(w)}}{\left\|e_{L}^{k}(w)\right\|_{h}}
$$

The density of states $\Pi_{h^{k}}(z)$ is the contraction of $\Pi_{k}(z, w)$ with the hermitian metric on the diagonal,

$$
\Pi_{h^{k}}(z):=\sum_{i=0}^{N_{k}}\left\|s_{i}^{k}(z)\right\|_{h_{k}}^{2}=F_{h^{k}}(z, z)|e(z)|_{h}^{2 k}=B_{k}(z, z),
$$

where in the first equality we record a standard abuse of notation in which the diagonal of the Szegö kernel is identified with its contraction. On the diagonal, we have the following asymptotic expansion the density of states,

$$
\Pi_{h^{k}}(z)=k^{m}\left(a_{0}+a_{1} S(z) k^{m-1}+a_{2}(z) k^{m-2}+\cdots\right)
$$

where $S(z)$ is the scalar curvature of $\omega$. The leading order term $a_{0}=1$ (see Section 1.8) if $\Pi_{h^{k}}(z, w)$ is the Szegö kernel relative to the volume form $d V=\frac{\omega^{m}}{m !}$.

1.7. Bergman kernels for a toric variety. In the case of a toric variety, we have

$$
F_{h^{k}}(z, w)=\sum_{\alpha \in k P \cap \mathbb{Z}^{m}} \frac{z^{\alpha} \bar{w}^{\alpha}}{Q_{h^{k}}(\alpha)},
$$

where $Q_{h^{k}}(\alpha)$ is defined in (9). If we sift out the $\alpha$ th term of $\Pi_{h^{k}}$ by means of Fourier analysis on $\mathbf{T}^{m}$, we obtain (2) .

Let $\widetilde{\varphi}(z, w)$ denote the almost extension of $\varphi(z)$ from the diagonal, that is $\widetilde{\varphi}$ satisfies the condition $\left.\bar{\partial}_{z}^{k} \widetilde{\varphi}(z, w)\right|_{z=w}=\left.\partial_{w}^{k} \widetilde{\varphi}(z, w)\right|_{z=w}=0$ for all $k \in \mathbb{N}$ and $\left.\widetilde{\varphi}(z, w)\right|_{z=w}=\varphi(z)$. The $\mathbf{T}^{m}$ action is by holomorphic isometries of $(M, \omega)$ and therefore

$$
\widetilde{\varphi}\left(\Phi^{\vec{t}} z, \Phi^{\vec{t}} w\right)=\widetilde{\varphi}(z, w)
$$

The Szegö kernel (17) admits a parametrix with complex phase $\widetilde{\varphi}$. In the case of a toric Kähler manifold, it takes the following simple form [STZ03].

Proposition 1.1. For any hermitian toric positive line bundle over a toric variety, the Szegö kernel for the metrics $h_{\varphi}^{N}$ have the asymptotic expansions in a local frame on $M$,

$$
B_{h^{k}}(z, w) \sim e^{k\left(\widetilde{\varphi}(z, w)-\frac{1}{2}(\varphi(z)+\varphi(w))\right)} A_{k}(z, w) \bmod k^{-\infty},
$$

where $A_{k}(z, w) \sim k^{m}\left(1+\frac{a_{1}(z, w)}{k}+\cdots\right)$ is a semi-classical symbol of order $m$ and where the phase satisfies (20).

1.8. Facts from algebraic geometry. If $L^{k}$ is very ample (i.e. the vanishing theorem holds), then $N_{k}:=\operatorname{dim} H^{0}\left(M, L^{k}\right)$ satisfies

$$
N_{k}+1=\chi\left(L^{k}\right)=\int_{M} e^{k c_{1}(L)} \operatorname{Td}(M)=a_{0} k^{m}+a_{1} k^{m-1}+\cdots+a_{m},
$$

where

$$
a_{0}=\frac{1}{m !} \int_{M} c_{1}(L)^{m}, \quad a_{1}=\frac{1}{(2(m-1) !} \int_{M} c_{1}(L)^{m-1} c_{1}(M)
$$

Also, $[\omega]=2 \pi c_{1}(L)$. Also

$$
\int_{M} \Pi_{h^{k}}(z, z) d V_{\omega}=\operatorname{dim} H^{0}\left(M, L^{k}\right)=a_{0} k^{m}+a_{1} k^{m-1}+\cdots,
$$

with

$$
a_{0}=\operatorname{Vol}_{\omega}(\mathrm{M})=\int_{\mathrm{M}} \frac{\omega^{\mathrm{m}}}{\mathrm{m} !}, \mathrm{a}_{1}=\frac{1}{2 \pi} \int_{\mathrm{M}} \mathrm{S}(\omega) \mathrm{dV}_{\omega}
$$

Here, $d V_{\omega}=\frac{\omega^{m}}{m !}=d \mu$. 
1.9. Asymptotic results on $\mathcal{Q}_{k}(\alpha)$ and $\mathcal{P}(\alpha, z)$. In [SoZ12, (23)], the norming constants are expressed in terms of the symplectic potential:

$$
\mathcal{Q}_{h^{k}}(\alpha)=\int_{P} e^{k\left(u_{0}(x)+\left\langle\frac{\alpha}{k}-x, \nabla u_{0}(x)\right\rangle\right.} d x .
$$

For interior $\alpha$, and $\alpha_{k}$ with $\left|\alpha-\alpha_{k}\right|=O\left(\frac{1}{k}\right)$,

$$
\mathcal{Q}_{h^{k}}\left(\alpha_{k}\right) \sim k^{-m / 2} e^{k u_{0}(\alpha)},
$$

and for all $\alpha$ and $\alpha_{k}$ with $\left|\alpha-\alpha_{k}\right|=O\left(\frac{1}{k}\right)$,

$$
\frac{1}{k} \log \mathcal{Q}_{h^{k}}\left(\alpha_{k}\right)=u_{0}(\alpha)+O\left(\frac{\log k}{k}\right) .
$$

The weights $\mathcal{P}_{h^{k}}(\alpha, z)(2)$ of the dilate $\mu_{k}^{z, 1}$ admit pointwise asymptotic expansions. The following is [ZZ18, Lemma 5.1].

LEMma 1.2. $\mathcal{P}_{h^{k}}(\alpha, z)=k^{m / 2}(2 \pi)^{-m / 2} \mid \operatorname{det} \operatorname{Hess}\left(\left.u_{\varphi}\left(\frac{\alpha}{k}\right)\right|^{\frac{1}{2}} e^{-k I^{z}\left(\frac{\alpha}{k}\right)}(1+O(1 / k))\right.$, where $O(1 / k)$ is uniform in $z, \alpha$. If $\left|\mu(z)-\frac{\alpha}{k}\right|=O\left(\frac{1}{k}\right)$, then

$$
\mathcal{P}_{h^{k}}(\alpha, z)=k^{m / 2}(2 \pi)^{-m / 2} \mid \operatorname{det} \operatorname{Hess}\left(\left.u_{\varphi}\left(\mu_{h}(z)\right)\right|^{\frac{1}{2}} e^{-k I^{z}\left(\frac{\alpha}{k}\right)}(1+O(1 / k)),\right.
$$

where $O(1 / k)$ is uniform in $z, \alpha$.

1.10. Probabilistic results. In [SoZ12] the following is proved:

Proposition 1.3. Let $(M, L, h, \omega)$ be a polarized toric Hermitian line bundle. Then the means, resp. variances of $\mu_{k}^{z}$ are given respectively by,

(1) $m_{k}(z)=\mu_{h}(z)+O\left(k^{-1}\right)$;

(2) $\Sigma_{k}(z)=k^{-1} \operatorname{Hess} \varphi+O\left(k^{-2}\right)$.

Moreover, the measures $\mu_{k}^{z}$ satisfy a weak law of large numbers; see (28).

Let $h=e^{-\varphi}$ be a toric Hermitian metric on $L$. Recall that the symplectic potential $u_{\varphi}$ associated to $\varphi$ is its Legendre transform: for $x \in P$ there is a unique $\rho(x)$ such that $\mu_{\varphi}\left(e^{\rho(x) / 2}\right)=d \varphi(\rho(x))=x$. If $z=e^{\rho / 2+i \theta}$ then we write $\rho_{z}=\rho=\log |z|^{2}$. Then the Legendre transform is defined to be the convex function

$$
u_{\varphi}(x)=\langle x, \rho(x)\rangle-\varphi(\rho(x)) .
$$

Also define

$$
I^{z}(x)=u_{\varphi}(x)-\left\langle x, \rho_{z}\right\rangle+\varphi\left(\rho_{z}\right) .
$$

Then $I^{z}(x)$ is a convex function on $P$ with a minimum of value 0 at $x=\mu_{h}(z)$ and with Hessian that of $u_{\varphi}$.

1.11. Large deviations. In [SoZ12] it is proved that the measures $\mu_{k}^{z}$ satisfy a large deviations principle with speed $k$ and a rate function $I^{z}$. The rate functions $I^{z}$ for $\left\{d \mu_{k}^{z}\right\}$ depend on whether $z$ lies in the open orbit $M^{o}$ of $M$ or on the divisor at infinity $\mathcal{D}$. The following is proved in [SoZ12].

TheOREM 1.4. For any $z \in M$, the probability measures $\mu_{k}^{z}$ satisfy a uniform Laplace large deviations principle with rate $k$ and with convex rate functions $I^{z} \geq 0$ on $P$ defined as follows:

- If $z \in M^{0}$, the open orbit, then $I^{z}(x)=u_{0}(x)-\langle x, \log |z|\rangle+\phi_{P^{\circ}}(z)$, where $\phi_{P^{\circ}}$ is the canonical Kähler potential of the open orbit and $u_{0}$ is its Legendre transform, the symplectic potential;

- When $z \in \mu_{0}^{-1}(F)$ for some face $F$ of $\partial P$, then $I^{z}(x)$ restricted to $x \in F$ is given by $I^{z}(x)=$ $u_{F}(x)-\left\langle x^{\prime}, \log \left|z^{\prime}\right|\right\rangle+\phi_{F}(z)$, where $\log \left|z^{\prime}\right|$ are orbit coordinates along $F, \phi_{F}$ is the canonical Kähler potential for the subtoric variety defined by $F$ and $u_{F}$ is its Legendre transform. On the complement of $\bar{F}$ it is defined to be $+\infty$.

- When $z$ is a fixed point then $I^{z}(v)=0$ and elsewhere $I^{z}(x)=\infty$.

The local asymptotics of Lemma 1.2 (due to [ZZ18, Lemma 5.1]) are derived from this large deviations principle. 
1.12. Bernstein polynomials and associated measures. One approach to entropy of the measures $\mu_{k}^{z}$ is to recognize their relation to Bernstein polynomials [Z09]. The Bernstein polynomials of a continuous function $f \in C(\bar{P})$ of a general toric Kähler manifold are quotients

$$
B_{h^{k}}(f)(x)=\frac{\mathcal{N}_{h^{k}} f(x)}{\Pi_{h^{k}}\left(\mu_{h}^{-1}(x), \mu_{h}^{-1}(x)\right)}
$$

of a numerator polynomial $\mathcal{N}_{h^{k}} f(x)$ by the denominator $\Pi_{h^{k}}(z, z)$ with $\mu_{h}(z)=x$. Here, $\mu_{h}$ is the moment map associated to the Kähler form $\omega_{h}$ associated to $h$, and

$$
\mathcal{N}_{h^{k}} f(x) \quad=\sum_{\alpha \in k P \cap \mathbb{Z}^{m}} f\left(\frac{\alpha}{k}\right) \frac{e^{k\left(u_{\phi}(x)+\left\langle\frac{\alpha}{k}-x, \nabla u_{\phi}(x)\right\rangle\right)}}{Q_{h^{k}}(\alpha)} .
$$

The numerator polynomials as well as the denominator admit complete asymptotic expansions: there exist differential operators $\mathcal{N}_{j}$, such that

$$
\mathcal{N}_{h^{k}}(f)(x) \sim \frac{k^{m}}{\pi^{m}}\left(f(x)+k^{-1} \mathcal{N}_{1} f(x)+\cdots\right),
$$

where the operators $\mathcal{N}_{j}$ are computable from the Bergman kernel expansion for $\Pi_{h^{k}}(z, z)$. In particular,

$$
\mathcal{N}_{1} f(x)=\frac{1}{2}\left(f(x) S\left(\mu_{h}^{-1}(x)\right)+\nabla \mu_{h}\left(\mu_{h}^{-1}(x)\right) \cdot \nabla^{2} f(x)\right),
$$

where $S(z)$ is the scalar curvature of the Kähler metric $\omega_{h}$. Combining the asymptotics of the numerator and denominator produces the asymptotics for $f \in C^{\infty}(\bar{P})$.

$$
B_{h^{k}}(f)(x)=f(x)+\mathcal{L}_{1} f(x) k^{-1}+\mathcal{L}_{2} f(x) k^{-2}+\cdots+\mathcal{L}_{m} f(x) k^{-m}+O\left(k^{-m-1}\right),
$$

in $C^{\infty}(\bar{P})$, where $\mathcal{L}_{j}$ is a differential operator of order $2 j$ depending only on curvature invariants of the metric $h$; the expansion may be differentiated any number of times.

The relevance of Bernstein polynomials to the measures $\mu_{k}^{z}$ is the following easily verifiable formula [Z09]: if $x=\mu_{\phi}(z)$ and let $h=e^{-\phi}$, then

$$
B_{h^{k}} f(x)=\int_{P} f(y) d \mu_{k}^{z}(y) .
$$

It follows that, for any $f \in C(\bar{P})$,

$$
\lim _{k \rightarrow \infty} \int_{P} f(y) d \mu_{k}^{z}(y)=f(\mu(z)) .
$$

\section{Proof of Theorem 0.2 on entropy asymptotics}

In this section, we prove Theorem 0.2. Since it is a rather technical calculation, we first give a detailed outline using prior results on the large deviations principle reviewed in Section 1.11, and on Bernstein polynomials (reviewed in Section 1.12). We then give a self-contained proof in Section 2.2. The outline is quite detailed and helps as a guide to the self-contained proof.

2.1. Sketch of proof. The entropy of $\mu_{k}^{z}$ is given explicitly in (4). The weights $\mathcal{P}_{h^{k}}(\alpha, z)(2)$ of the dilate $\mu_{k}^{z, 1}$ admit pointwise asymptotic expansions in Lemma 1.2. We assume that $\mu(z) \in P^{o}$, so that $I^{z}(x)=u_{0}(x)-\langle x, \log |z|\rangle+\phi_{P^{o}}(z)$. Unravelling the logarithm in (4) gives,

$$
H\left(\mu_{k}^{z}\right)=-\frac{1}{\Pi_{h^{k}}(z, z)} \sum_{\alpha \in k P \cap \mathbb{Z}^{m}} \mathcal{P}_{h^{k}}(\alpha, z)\left(\log \mathcal{P}_{h^{k}}(\alpha, z)-\log \Pi_{h^{k}}(z, z)\right) .
$$

By (18),

$$
\text { (I) } \frac{1}{\Pi_{h^{k}}(z, z)} \sum_{\alpha \in k P \cap \mathbb{Z}^{m}} \mathcal{P}_{h^{k}}(\alpha, z) \log \Pi_{h^{k}}(z, z) \sim \log \left(a_{0} k^{m}\right)+O\left(\frac{1}{k}\right)=m \log k+O\left(\frac{1}{k}\right) .
$$

Therefore it suffices to determine the asymptotics of the first term of (29),

$$
-\frac{1}{\Pi_{h^{k}}(z, z)} \sum_{\alpha \in k P \cap \mathbb{Z}^{m}} \mathcal{P}_{h^{k}}(\alpha, z) \log \mathcal{P}_{h^{k}}(\alpha, z) .
$$

By Lemma 1.2,

$$
\log \mathcal{P}_{h^{k}}(\alpha, z)=\log \left(k^{m / 2}(2 \pi)^{-m / 2}\right)+\log \mid \operatorname{det} \operatorname{Hess}\left(\left.u_{\varphi}\left(\mu_{h}(z)\right)\right|^{\frac{1}{2}}-k I^{z}\left(\frac{\alpha}{k}\right)+O(1 / k)\right) .
$$


Since the first two terms are independent of $\alpha$, we obtain a second term,

$$
\begin{aligned}
& (I I)-\frac{1}{\Pi_{h^{k}}(z, z)} \sum_{\alpha \in k P \cap \mathbb{Z}^{m}} \mathcal{P}_{h^{k}}(\alpha, z)\left(\log \left(k^{m / 2}(2 \pi)^{-m / 2}\right)+\log \mid \operatorname{det} \operatorname{Hess}\left(\left.u_{\varphi}\left(\mu_{h}(z)\right)\right|^{\frac{1}{2}}\right)\right. \\
& =-\log \left(k^{m / 2}(2 \pi)^{-m / 2}\right)-\log \left|\operatorname{det} \operatorname{Hess}\left(u_{\varphi}\left(\mu_{h}(z)\right)\right)\right|^{\frac{1}{2}}+O\left(\frac{1}{k}\right) .
\end{aligned}
$$

Thus, we are left with the third term,

$$
(I I I) \frac{1}{\Pi_{h^{k}}(z, z)} \sum_{\alpha \in k P \cap \mathbb{Z}^{m}} \mathcal{P}_{h^{k}}(\alpha, z)\left(k I^{z}\left(\frac{\alpha}{k}\right)\right) .
$$

We obtain asymptotics for this term using the asymptotics of Bernstein polynomials as reviewed above . To make this connection, we define a function $f_{z}$ so that

$$
f_{z}\left(\frac{\alpha}{k}\right)=I^{z}\left(\frac{\alpha}{k}\right)
$$

Both sides extend with no complication from the lattice points $\frac{\alpha}{k}$ to all $x \in P^{o}$. By Theorem 1.4, it follows that term (III) is, up to errors of order $O\left(\frac{1}{k}\right)$, the Bernstein polynomial for

$$
f_{z}(x)=I^{z}(x)=u_{0}(x)-\langle x, \log |z|\rangle+\phi_{P^{o}}(z) .
$$

Note that since $u$ and $\phi$ are Legendre transforms, one has

$$
u(x)+\phi(\rho)=\langle x, \rho\rangle, \quad x=\mu\left(e^{\rho}\right) .
$$

By (27), the leading term in the asymptotic expansion of $(I I I)$ is 0 . Since this term is multiplied by $k$, this signals that $(I I I)=O(1)$. Since $f(\mu(z))=0$, the leading order asymptotics is given by the second term,

$$
\left(\text { III.0) } \mathcal{N}_{1} f(x)=\frac{1}{2}\left(f(x) S\left(\mu_{h}^{-1}(x)\right)+\nabla \mu_{h}\left(\mu_{h}^{-1}(x)\right) \cdot \nabla^{2} f(x)\right)=\nabla \mu_{h}\left(\mu_{h}^{-1}(x)\right) \cdot \nabla^{2} I^{z}(\mu(z)) .\right.
$$

However, $\nabla^{2} I^{z}=\nabla^{2} u_{0}$, so the last term is $\operatorname{Tr}\left(\mathrm{I}_{\mathrm{m}}\right)=\mathrm{m}$.

Adding the contributions of (30)-(32)-(34) gives

$$
\begin{aligned}
H\left(\mu_{k}^{z}\right) & =(I)+(I I)+(I I I .0) \\
& =\log \left(k^{m}\right)-\log \left(k^{m / 2}(2 \pi)^{-m / 2}\right)-\log \mid \operatorname{det} \operatorname{Hess}\left(\left.u_{\varphi}\left(\mu_{h}(z)\right)\right|^{\frac{1}{2}}+\frac{m}{2}+O\left(\frac{1}{k}\right),\right. \\
& =\frac{m}{2}(1+\log (2 \pi k))-\log \mid \operatorname{det} \operatorname{Hess}\left(\left.u_{\varphi}\left(\mu_{h}(z)\right)\right|^{\frac{1}{2}}+O\left(\frac{1}{k}\right)\right.
\end{aligned}
$$

agreeing with the formula of Theorem 0.2.

REMARK 6. Above, we used that $a_{0}=1$ to simplify the first term.

2.2. A more detailed proof. We now give a more detailed proof without using prior results on Bernstein polynomials.

Let $\tilde{Q}_{k}(y):=e^{k u_{0}(y)} \int_{P} e^{k\left(u_{0}(x)-u_{0}(y)+<\nabla u_{0}(x), y-x>\right)} d x$

Lemma 2.1. For all neighborhoods of the boundary of the polytope $U$ we have a uniform equivalent outside of $U, \tilde{Q}_{k}(y)=(2 \pi)^{m / 2}\left|\operatorname{det} \nabla^{2} u_{0}(y)\right|^{-1 / 2} k^{m / 2} e^{k u_{0}(y)}\left(1+\frac{m_{k}(y)}{k^{1 / 2}-\epsilon}\right)$ where $\sup _{k \in \mathbb{N}, y \in P-U}\left|m_{k}(y)\right|<\infty$

Proof. let $f_{y}(x)=u_{0}(y)-u_{0}(x)+\left\langle\nabla u_{0}(x), x-y\right\rangle$. It is a positive function that attain 0 only once in $y$ and whose Hessian is $\left.\nabla^{2} u_{0}\right|_{y}$ at $y$.

$$
\begin{aligned}
\tilde{Q}_{k}(y) & =e^{k u_{0}(y)} \int_{P} e^{-k f_{y}(x)} d x \\
& =e^{k u_{0}(y)}\left(\int_{B\left(y, \delta_{k}\right)} e^{-k\left(\left.\nabla^{2} u_{0}\right|_{y}(x-y, x-y)+\delta f(x)\right)} d x+K e^{-k \inf _{P-B\left(x, \delta_{k}\right)} f_{y}(x)}\right) .
\end{aligned}
$$

Let $m_{y}^{\delta}:=\frac{\inf _{P-B(x, \delta)} f_{y}(x)}{2 \delta^{2}}$ and $M_{y}^{\delta}:=\frac{f_{y}(x)-\nabla^{2} u_{0}(x-y, x-y)}{6 \delta^{3}}$ 
We have two bounds on $\tilde{Q}_{k}(y)$

$$
\begin{aligned}
\int_{B\left(y, \delta_{k}\right)} e^{-\frac{k}{2} \nabla^{2} u_{0}(x-y, x-y)-k \delta_{k}^{3} M_{y}^{\delta_{k}}} d x \leq \tilde{Q}_{k}(y) e^{-k u_{0}(y)} & \leq \int_{B\left(y, \delta_{k}\right)} e^{-\frac{k}{2} \nabla^{2} u_{0}(x-y, x-y)+k \delta_{k}^{3} M_{y}^{\delta_{k}}} d x \\
& +\operatorname{Vol}(P) e^{-k \delta_{k}^{2} m_{y}^{\delta_{k}}}
\end{aligned}
$$

Now changing the variables in the integral leads to

$$
\begin{aligned}
\int_{B\left(0, \sqrt{k} \delta_{k}\right)} e^{-\frac{1}{2} \nabla^{2} u_{0}(z, z)-k \delta_{k}^{3} M_{y}^{\delta_{k}}} k^{m / 2} d z \leq & \tilde{Q}_{k}(y) e^{-k u_{0}(y)} \leq \int_{B\left(0, \sqrt{k} \delta_{k}\right)} e^{-\frac{1}{2} \nabla^{2} u_{0}(z, z)+k \delta_{k}^{3} M_{y}^{\delta_{k}}} k^{m / 2} d z \\
& +\operatorname{Vol}(P) e^{-k \delta_{k}^{2} m_{y}^{\delta_{k}}}
\end{aligned}
$$

In order for the whole term to converge we need to choose $\delta_{k}$ to carefully. If we choose $\delta_{k}=\epsilon k^{-\alpha}$ with $\alpha \in\left(\frac{1}{3}, \frac{1}{2}\right)$ we'll obtain a exponential rate of convergence.

More over as $k$ goes to infinity, $\int_{B\left(0, \sqrt{k} \delta_{k}\right)} e^{-\frac{1}{2} \nabla^{2} u_{0}(z, z)}=\sqrt{\operatorname{det} 2 \pi\left(\nabla^{2} u_{0}\right)^{-1}}\left(1+N_{k}\left(\sqrt{k} \delta_{k}\right)^{m-2} e^{-\frac{\left(\sqrt{k} \delta_{k}\right)^{2}}{2}}\right)$ with $\left(N_{k}\right)_{k \in \mathbb{N}}$ a bounded sequence.

Now we have the following sandwich :

$$
\begin{aligned}
e^{-k \delta_{k}^{3} M_{y}^{\delta_{k}}(1+} & \left.N_{k}\left(\sqrt{k} \delta_{k}\right)^{m-2} e^{-\frac{\left(\sqrt{k} \delta_{k}\right)^{2}}{2}}\right) \leq \\
\frac{Q_{k}(y) \sqrt{\operatorname{det} \nabla^{2} u_{0}(y)}}{(2 \pi)^{\frac{m}{2}} k^{m / 2} e^{k u_{0}(y)}} & \leq e^{k \delta_{k}^{3} M_{y}^{\delta_{k}}}\left(1+N_{k}\left(\sqrt{k} \delta_{k}\right)^{m-2} e^{-\frac{\left(\sqrt{k} \delta_{k}\right)^{2}}{2}}+N_{k} k^{-\frac{m}{2}} e^{-k \delta_{k}^{2} m_{y}^{\delta_{k}}}\right)
\end{aligned}
$$

The first vanishing term comes from the term in $e^{k \delta_{k}^{3} M_{y}^{\delta_{k}}}$ The final equivalent is of the form :

$$
\frac{Q_{k}(y) \sqrt{\operatorname{det} \nabla^{2} u_{0}(y)}}{(2 \pi)^{\frac{m}{2}} k^{m / 2} e^{k u_{0}(y)}}=1+c_{k}^{y} k \delta_{k}^{3}=1+c_{k}^{y} k^{1-3 \alpha}=1+c_{k}^{y} k^{-\frac{1}{2}+\epsilon}
$$

With $\left(c_{k}^{y}\right)_{k \in \mathbb{N}}$ a bounded sequence. In order to prove that $\left(c_{k}^{y}\right)_{k \in \mathbb{N}}$ is uniformly bounded over $P-U$, we just need to show that $m_{y}^{\delta}$ and $M_{y}^{\delta}$ are uniformly bounded. For any neighborhood $U$ of the boundary of $P$, $P-U$ is a compact set where $u_{0}$ is $C^{\infty}$ and so where $m_{y}^{\delta}$ and $M_{y}^{\delta}$ are uniformly bounded.

2.3. Computation of the entropy. Now that we proved this technical lemma, we'll use it and the asymptotics of the Szegö kernel $\Pi(z, z)=k^{m}+O\left(k^{m-1}\right)$ to obtain the following uniform asymptotic for the individual probabilities of the sequence of measures : Let's take any neighborhood of the boundary $U$ such that $\mu(z)$ is in the interior of $P-U$. We have that $\forall \alpha \in P-U$ such that $k \alpha \in k P \cap \mathbb{Z}^{m}$ for a certain $k$.

$$
\mu_{k}^{z}(\alpha)=\frac{\left|z^{\alpha}\right|_{h^{k}}^{2}}{Q_{k}(\alpha) \Pi_{k}(z, z)}=\frac{\sqrt{\operatorname{det} \nabla^{2} u_{0}(y)}}{(2 \pi k)^{-m / 2}} e^{-k I^{z}(\alpha)}\left(1+\frac{c_{\alpha}}{k^{1 / 2-\epsilon}}\right)
$$

With $\left|c_{\alpha}\right| \leq M$ and $M$ only depending on $U$.

Let's split the calculation in two :

$$
H\left(\mu_{k}^{z}\right)=-\sum_{p \in U} \mu_{k}^{z}(p) \log \left(\mu_{k}^{z}(p)\right)-\sum_{p \in P-U} \mu_{k}^{z}(p) \log \left(\mu_{k}^{z}(p)\right)
$$

Lemma 2.2. The first term goes to zero

Proof. Let $\nu_{k}:=\frac{\mu_{k}^{z}(\cdot) \mathbf{1} \in U}{\mu_{k}^{z}(U)}$. Then

$$
-\sum_{p \in U} \mu_{k}^{z}(p) \log \left(\mu_{k}^{z}(p)\right)=-\mu_{k}^{z}(U) \log \left(\mu_{k}^{z}(U)\right)+\mu_{k}^{z}(U) H\left(\nu_{k}\right)
$$

Except that $\nu$ is concentrated on a finite number of points that is equal to $|U| k^{m}+o\left(k^{d}\right)$, meaning that $H\left(\nu_{k}\right) \leq d \log (k)+$ constant and $\mu_{k}^{z}(U)$ decrease exponentially due to the LDP. This implies that the result.

$$
-\sum_{p \in U} \mu_{k}^{z}(p) \log \left(\mu_{k}^{z}(p)\right) \rightarrow_{k \rightarrow \infty} 0
$$


We need to compute the second term $H^{\prime}\left(\mu_{k}^{z}\right)$. Let's split the sum again in four parts. We'll use the notation $P_{k}^{U}:=\left(k P \cap \mathbb{Z}^{m}\right) / k-U$.

$$
\begin{aligned}
H^{\prime}\left(\mu_{k}^{z}\right): & =\sum_{\alpha \in P_{k}^{U}} \mu_{k}^{z}(\alpha) \log \left((2 \pi k)^{m / 2}\right) \\
& -\sum_{\alpha \in P_{k}^{U}} \mu_{k}^{z}(\alpha) \log \left(\sqrt{\operatorname{det} \nabla^{2} u_{0}(\alpha)}\right) \\
& +\sum_{\alpha \in P_{k}^{U}} \mu_{k}^{z}(\alpha) k I^{z}(\alpha) \\
& -\sum_{\alpha \in P_{k}^{U}} \mu_{k}^{z}(\alpha) \log \left(1+\frac{c_{\alpha}}{k^{1 / 2-\epsilon}}\right)
\end{aligned}
$$

Then trivially we have that $|(4)|<\log \left(1+\frac{M}{k^{1 / 2-\epsilon}}\right) \underset{k \rightarrow \infty}{\rightarrow} 0$ and that $(1)=\frac{m}{2} \log (2 \pi k)+\mathrm{o}(1)$.

For (2) we need to notice that $u_{0}$ is strictly convex on the interior of $P$, so it's stricly concave on $P-U$. $\log \left(\left|\operatorname{det} \nabla^{2} u_{0}(\alpha)\right|\right)^{1 / 2}$ is a consequently a continuous bounded function on $P-U$ and the LDP implies that the term converges to $\log \left(\left|\operatorname{det} \nabla^{2} u_{0}(\mu(z))\right|^{1 / 2}\right)$.

The only difficult term to compute is the third, which we'll denote $A_{3}$.

Lemma 2.3. $\lim _{k \rightarrow \infty} A_{3}=\frac{m}{2}$

Proof. Let $K:=-\nabla^{2} u_{0}(\mu(z))$. For a $\delta$ arbitrary small, we have :

- Outside of $B_{\delta}\left(x_{0}\right)$ we have $I^{z}>\epsilon_{1}(\delta) \delta^{2}$ with $\epsilon_{1}(\cdot)$ a strictly positive function with a strictly positive lower bound

- Inside of $B_{\delta}\left(x_{0}\right)$ we have $f_{k}(\alpha)=\left|\operatorname{det} \nabla^{2} u_{0}(\alpha)\right|^{1 / 2}\left(1+\frac{c_{\alpha}^{k}}{k^{1 / 2-\epsilon}}\right)=|\operatorname{det} K|^{1 / 2}\left(1+\epsilon_{2}^{k}\left(\alpha-x_{0}\right)\right)$ such that $\left\|\epsilon_{2}^{k}(\cdot)\right\|_{\infty}<\epsilon_{2, \delta}^{k}$, with $\epsilon_{2, \delta}^{k}$ increasing with $\delta$ and decreasing with $k$ such that it vanishes as $k \rightarrow \infty$ and $\delta \rightarrow 0$.

- Inside of $B_{\delta}\left(x_{0}\right)$ we also have that $I^{z}\left(x_{0}+\delta x\right)=\frac{1}{2} K(\delta x, \delta x)+\epsilon_{3}(\delta x)\|\delta x\|^{3}$ with $\left\|\epsilon_{3}(\cdot)\right\|_{\infty}<\infty$

We treat the $\epsilon_{1}$ as an increasing positives functions of $\delta$ that vanish in 0 and $\epsilon_{2}^{k}$ as an increasing with $\delta$ and decreasing with $k$ positive function that vanish in 0 as $k \rightarrow \infty$. We then have :

$$
\begin{aligned}
\frac{A_{3}}{\sqrt{\operatorname{det} K}(2 \pi)^{-m / 2}} & =\frac{(2 \pi)^{-m / 2}}{\sqrt{\operatorname{det} K}} \sum_{\alpha \in P_{k}^{U}} \mu_{k}^{z}(\alpha) k I^{z}(\alpha) \\
& =\sum_{\alpha \in P_{k}^{U} \cap B_{\delta}\left(x_{0}\right)} k^{-m / 2} \sqrt{\frac{\left|\operatorname{det} \nabla^{2} u_{0}(\alpha)\right|}{\operatorname{det} K}}\left(1+\frac{c_{\alpha}}{k^{1 / 2-\epsilon}}\right) e^{-k I^{z}(\alpha)} k I^{z}(\alpha)+O\left(k^{1+m / 2} e^{-k \epsilon_{1}(\delta) \delta^{2}}\right) \\
& =k^{-m / 2} \sum_{\delta x \in\left(P_{k}^{U}-x_{0}\right) \cap B_{\delta}(0)} e^{-\frac{k}{2} K(\delta x, \delta x)} \frac{k}{2} K(\delta x, \delta x)\left(1+O\left(\epsilon_{2}^{k}\right)+O\left(k \delta^{3}\right)\right)+O\left(k^{1+m / 2} e^{-k \epsilon_{1}(\delta) \delta^{2}}\right)
\end{aligned}
$$

with the two $O$ being uniformly bounded over $\left(P_{k}^{U}-x_{0}\right) \cap B_{\delta_{0}}(0)$. Now let's scale up the sum.

$$
=k^{-m / 2} \sum_{\delta x \in \sqrt{k}\left(P_{k}^{U}-x_{0}\right) \cap B_{\sqrt{k} \delta}(0)} e^{-\frac{1}{2} K(\delta x, \delta x)} \frac{1}{2} K(\delta x, \delta x)\left(1+O\left(\epsilon_{2}^{k}\right)+O\left(k \delta^{3}\right)\right)+O\left(k^{1+m / 2} e^{-k \epsilon_{1}(\delta) \delta^{2}}\right)
$$


Now the set $P_{\delta}^{k}:=\sqrt{k}\left(P_{k}^{U}-x_{0}\right) \cap B_{\sqrt{k} \delta}(0)$ is for small enough $\delta$ and interior $x_{0}$ the set $\frac{1}{\sqrt{k}}\left(\mathbb{Z}^{m}-x_{0}\right) \cap B_{\sqrt{k} \delta}$. If we choose a specific sequence of $\delta_{k}=\frac{\epsilon}{k \gamma}$ with $\gamma \in\left(\frac{1}{3}, \frac{1}{2}\right)$, the series converges as $k$ goes to infinity and all the $O$ s vanish from the limit.

The series is a truncated step function approximation of the following integral

$$
\int_{\mathbb{R}^{m}} \frac{K(x, x)}{2} e^{-\frac{K(x, x)}{2}} d x=\sqrt{\operatorname{det} \frac{2 \pi}{K}} \frac{m}{2}
$$

Which is Riemann integrable so we don't need further arguments. Finally,

$$
\lim _{k \rightarrow \infty} A_{3}=\frac{m}{2 a_{0}(2 \pi)^{m}}
$$

By binding all the pieces together, we obtain the following asymptotics for the entropy of the measures $\mu_{k}^{z}$ :

$$
H\left(\mu_{k}^{z}\right) \underset{k \rightarrow \infty}{=} \frac{m}{2} \log (2 \pi k)-\frac{1}{2} \log \left(\left|\operatorname{det} \nabla^{2} u_{0}\left(x_{0}\right)\right|\right)+\frac{m}{2}+o(1)
$$

This concludes the proof of Theorem 0.2.

2.4. Point $z$ for which $\mu_{k}^{z}$ has asymptotically maximal entropy: Proof of Theorem 0.6. We now consider the point $x=\mu(z)$ for which the measure $\mu_{k}^{z}$ has asymptotically maximal entropy within the family $\left\{\mu_{k}^{z}\right\}$. For Fano Kähler manifolds, we prove that there exists a unique point $x=\mu(z)$ at which $\mu_{k}^{z}$ has asymptotically maximal entropy. For Fano Kähler manifolds with Kähler -Einstein metric, we prove that the unique point $x \in P$ is the point $x=0$.

Proof. As mentioned in the introduction, it follows from Theorem 0.2 that the points $z$ such that $\mu_{k}^{z}$ has asymptotically maximal entropy are points where the Ricci potential is maximal. Due to the inverse relation of det $i \partial \bar{\partial} \phi$ and $L(x)=\log \left(\left|\operatorname{det} \nabla^{2} u_{0}\left(x_{0}\right)\right|\right)(5)$, points where the Ricci potential is maximal lie in the inverse image under the moment map $\mu_{h}$ of points $x$ for which $L(x)$ is minimal (see Theorem 0.3). Since $u$ is convex, we may remove the absolute value.

It is proved in [Ab98, Theorem 2.8] that $L(x)$ is a smooth function on the interior of $P$ and $L(x) \uparrow \infty$ as $x \rightarrow \partial P$. Consequently, $u$ has a global minimum which lies in the interior of $P$. This proves existence for all toric Kähler manifolds.

If we assume that Ricci curvature $\operatorname{Ric}(\omega)$ is positive definite, then the minimum is unique. Indeed, by (6), positive Ricci curvature is equivalent to $L(x)$ (5) being a strictly convex function. Since $L(x) \uparrow \infty$ as $x \rightarrow \partial P, L(x)$ is proper and strict convexity implies that the minimum of $u$ is unique. We state the result as the following,

Lemma 2.4. If $(M, \omega)$ is a toric Fano manifold of positive Ricci curvature, then there is a unique point $\rho_{0}$ in the open orbit of maximal entropy, corresponding to a unique point $x_{0}=\mu_{h}\left(e^{\rho_{0} / 2}\right) \in P$.

We now assume further that $\omega$ is a toric Kähler -Einstein metric. In that case, the equation $\operatorname{Ric}(\omega)=a \omega$ (for some $C>0$ ) implies that there exists a constant vector $\vec{b}$ and $c \in \mathbb{R}$ so that

$$
-\log \operatorname{det}(i \partial \bar{\partial} \varphi)(\rho)=a \varphi(\rho)+\vec{b} \cdot \rho+c .
$$

Indeed, $\partial \bar{\partial}(-\log \operatorname{det}(i \partial \bar{\partial} \varphi)(\rho)-a \varphi(\rho))=0$ and therefore the difference potential is a toric pluriharmonic function, hence a linear function. By Lemma 2.4, we get

Lemma 2.5. If If $(M, \omega)$ is a toric Kähler-Einstein Fano manifold of positive Ricci curvature, then in the gauge (47), there is a unique critical point in the open orbit where

$$
\nabla \varphi(\rho)=\mu_{h}\left(e^{\rho}\right)=-\frac{1}{a} \vec{b}
$$


Alternatively, we may write the critical point equation in terms of the symplectic potential $u$ and the function $L(x)(5)$. The Kähler -Einstein equation (47) for the potentials then changes to,

$$
L(x)=a \phi\left(e^{\rho_{x}}\right)+\vec{b} \cdot \rho_{x}+c, \quad \mu_{h}\left(\rho_{x}\right)=x .
$$

Since $u=\mathcal{L} \phi$ (Legendre transform), and $\nabla u(x)=\mu_{h}^{-1}(x)$ one has $u(x)=x \cdot \rho_{x}-\phi\left(e^{\rho_{x}}\right)$ and so (49) simplifies to,

$$
L(x)=a(x \cdot \nabla u(x)-u(x))+\vec{b} \cdot \nabla u(x)+c=(a x+\vec{b}) \cdot \nabla u-a u(x)+c .
$$

As mentioned in Section 1.3, toric Kähler potentials are not unique because of the gauge symmetries. For instance, one may add a linear function of $\rho$ to obtain an equivalent potential. The shift of gauge by an affine function results in a translation of the Delzant polytope. According to [M87, Definition 3.6], there exists an open orbit toric Kähler potential $\phi$ so that so that

$$
\operatorname{det} D_{\rho}^{2} \varphi=e^{-\varphi}, \quad e^{-\varphi} \prod_{j=1}^{m} \frac{i d z_{j} \wedge d \bar{z}_{j}}{\left|z_{j}\right|^{2}} \in C^{\infty}(M, \Omega),
$$

where $z_{j}$ are open orbit coordinates (in $\left(\mathbb{C}^{*}\right)^{m}$ and $C^{\infty}(M, \Omega)$ is the space of smooth volume forms. We refer to (51) as the Mabuchi or Kähler -Einstein gauge. In the gauge (51), the the potential satisfies,

$$
\varphi=-\log \operatorname{det}(i \partial \bar{\partial} \varphi)(\rho)(\vec{b}=0) .
$$

Combining with Lemma 2.5 gives,

Lemma 2.6. In the gauge (52) for the Kähler potential, the unique point of maximal entropy solves the critical point equation,

$$
\nabla_{\rho} \varphi(\rho)=0, \quad\left(\Longleftrightarrow \mu\left(e^{\rho / 2}\right)=0\right) .
$$

It follows that in this gauge, the point $x_{0} \in P$ for which $\mu_{k}^{z_{0}}$ has asymptotically maximal entropy is the origin $0 \in P$.

This completes the proof of Theorem 0.6.

REMARK 7. According to [M87], the origin in the gauge (51) is the center of mass of the polytope P. This follows from the facts that, by [M87, Corollary 5.5], the so-called Futaki invariant $\mathbf{a}_{P}=0$, and by [M87, Theorem 9.2.3], that the center of mass of $P$ is 0.

One may directly prove that the center of mass in the Kähler-Einstein gauge equals 0 , i.e. the gauge $\varphi$ such that $-\log \operatorname{det} i \partial \bar{\partial} \varphi=a \varphi$, using the moment map change of variables $\mu_{h}\left(e^{\rho}\right)=\nabla_{\rho} \varphi(\rho)=x(\rho)$, which gives

$$
x_{\text {mass }}=\frac{1}{\operatorname{Vol}(P)} \int_{P} x d x=\frac{1}{\operatorname{Vol}(P)} \int_{\mathbb{R}^{n}} \nabla_{\rho} \varphi(\rho) \operatorname{det} \nabla_{\rho}^{2} \varphi d \rho .
$$

Using the fact that $\operatorname{det} i \partial \bar{\partial} \varphi\left(e^{\rho}\right)=\operatorname{det} \nabla_{\rho}^{2} \varphi d \rho=e^{-\varphi(\rho)} d \rho$ we get that

$$
x_{\text {mass }}=-\frac{1}{\operatorname{Vol}(P)} \int_{\mathbb{R}^{n}} \nabla_{\rho}\left(e^{-\varphi}\right) d \rho
$$

By (10), $\varphi(\rho)=\max _{\alpha \in P \cap \mathbb{Z}^{m}} \rho \cdot \alpha+O(1)$ as $\rho \rightarrow \infty$. As long as $\max _{\alpha \in P \cap \mathbb{Z}^{m}}(\rho \cdot \alpha) \geq \epsilon|\rho|$ for some $\epsilon>0$ and large $\rho, e^{-\varphi}$ is rapidly decreasing and one can integrate by parts in (53) to prove that $x_{\text {mass }}=0$. The lower bound is true if and only if $0 \in P^{o}$. It must be the case that $\max _{\alpha \in P \cap \mathbb{Z}^{m}} \rho \cdot \alpha \geq \epsilon|\rho|$ for large $\rho$; otherwise, there exists direction in which $\varphi$ does not go to $+\infty$ implying that

$$
\operatorname{Vol}(P)=\int_{P} d x=\int_{\mathbb{R}^{n}} e^{-\varphi} d \rho=\infty
$$

which is a contradiction thus proving the result.

Some further references for the existence of the potential satisfying (51) are [WZ04, W15]. The formula (50) agrees with $[W Z 04,(2.18)]$. They define the parameters $c_{\ell}$ by,

$$
\int_{P} y_{\ell} \exp \left\{\sum_{\ell=1}^{n} c_{\ell} y_{\ell}\right\} d y=0 .
$$


if $M$ admits a Kähler -Einstein metric then $\vec{c}=0$; in terms of our notation, $\vec{c}$ is the center of mass of $P$. See also [W15, Page 3615] and [D08, Section 3.3].

Let us check the identities in the simplest case of $\mathbb{C P}^{1}$ with Fubini-Study metric. If we choose the gauge $u_{F S}(x)=x \log x+(1-x) \log (1-x)$ in which $P=[0,1]$, then $\log \left(u_{F S}^{\prime \prime}(x)\right)^{-1}=\log x(1-x), b=1, a=-2$. Similar equations hold for $\mathbb{C P}^{m}$. If we choose a gauge for which $u_{r}(x)=\frac{1}{2}\left(\left(r^{2}+x\right) \log \left(r^{2}+x\right)+\left(r^{2}-x\right) \log \left(r^{2}-x\right)\right)$ and $P=\left[-r^{2}, r^{2}\right]$, then $b=0$.

\section{Convolution powers And toric measure: Proof of Theorem 0.4}

3.1. Proof of Proposition 0.5. First, we prove Proposition 0.5. For the reader's convenience we recall that it says that, for any Kähler manifold $(M, \omega, J)$, the following are equivalent:

(1) $\operatorname{Hilb}_{\mathrm{k}}(\mathrm{h})$ is balanced for all $k$, i.e. there exist constants $C_{k}$ so that the density of states $\Pi_{h^{k}}(z)=C_{k}$ for all $k$.

(2) $\Pi_{h^{k}}(z, w)=A_{k}\left(\Pi_{h^{1}}(z, w)\right)^{k}$, where

$$
\frac{\operatorname{dim} H^{0}\left(M, L^{k}\right)}{\operatorname{vol}(\mathrm{M}, \omega)}=A_{k}\left(\frac{\operatorname{dim} H^{0}(M, L)}{\operatorname{vol}(\mathrm{M}, \omega)}\right)^{k} .
$$

Proof. (1) $\Longrightarrow(2)$. If $\Pi_{h^{k}}(z, z)=C_{k}$ for some constant $C_{k}$, then necessarily $C_{k}=\frac{\operatorname{dim} H^{0}\left(M, L^{k}\right)}{\operatorname{vol}(\mathrm{M}, \omega)}$. If (1) holds, then the constant $C_{k}$ in (1) is given by this formula for all $k$. We then define $A_{k}$ by $A_{k}=\frac{C_{k}}{C_{1}^{k}}$. Thus, $C_{k}$ and $A_{k}$ are uniquely determined by the assumption (1) and by definition of $A_{k}$ we have $\Pi_{h^{k}}(z, z)=$ $A_{k}\left(\Pi_{h^{1}}(z, z)\right)^{k}$. This equation holds along the totally real submanifold $\{(z, \bar{z}): z \in M\} \subset M \times \bar{M}$, where we identify the complexification of $M$ with $M \times \bar{M}$. Since $\Pi_{h^{k}}(z, w)$ is holomorphic, the equation implies $\Pi_{h^{k}}(z, w)=A_{k}\left(\Pi_{h^{1}}(z, w)\right)^{k}$ for all $(z, w) \in M \times \bar{M}$, i.e. (1) $\Longrightarrow(2)$.

$(2) \Longrightarrow(1)$. Conversely assume (2). To prove (1) it suffices to prove that $\Pi_{h^{1}}(z, z)=C_{1}$. If this is false, we introduce constants $\alpha<1, \beta>1$ and consider the sets $M_{-}=\left\{z: \Pi_{h^{1}}(z, z)<\alpha C_{1}\right\}$ and $M_{+}=\left\{z: \Pi_{h^{1}}(z, z)>\beta C_{1}\right\}$. If $\Pi_{h^{1}}(z, z)=C_{1}$ is false, $M_{ \pm}$must be non-empty open sets for some $\alpha<1, \beta>1$. Assuming (2), we have $\Pi_{h^{k}}(z, z)<A_{k} C_{1}^{k} \alpha^{k}$ in $M_{-}$and $\Pi_{h^{k}}(z, z)>A_{k} C_{1}^{k} \beta^{k}$ in $M_{+}$. But $A_{k} C_{1}^{k}=C_{k}=\frac{1}{\operatorname{Vol}(\mathrm{M})} \operatorname{dim} H^{0}\left(M, L^{k}\right)$, and standard Bergman kernel asymptotics give $\Pi_{h^{k}}(z, z) \simeq$ $\frac{1}{\operatorname{Vol}(\mathrm{M})} \operatorname{dim} H^{0}\left(M, L^{k}\right)+o\left(\operatorname{dim} H^{0}\left(M, L^{k}\right)\right)$. We then get the contradiction that $\frac{1}{\operatorname{Vol}(\mathrm{M})} \operatorname{dim} H^{0}\left(M, L^{k}\right)<$ $\alpha^{k} \frac{1}{\operatorname{Vol}(\mathrm{M})} \operatorname{dim} H^{0}\left(M, L^{k}\right)$ in $M_{-}$and $\frac{1}{\operatorname{Vol}(\mathrm{M})} \operatorname{dim} H^{0}\left(M, L^{k}\right)>\beta^{k} \frac{1}{\operatorname{Vol}(\mathrm{M})} \operatorname{dim} H^{0}\left(M, L^{k}\right)$, concluding the proof.

REMARK 8. We note that $\Pi_{h^{k}}(z, w)$ is holomorphic in $z$ and anti-holomorphic in $w$. The density of states $\rho_{k}=\Pi_{h^{k}}(z, z)$ is the metric contraction of the diagonal values of $\Pi_{h^{k}}(z, w)$ by the metric $e^{-k \phi(z)}$ and therefore is not the restriction of a holomorphic function on the complexification $M \times \bar{M}$ to the anti-diagonal. Hence, $\Pi_{h^{k}}(z, z)=C_{k}$ does not imply that $\Pi_{h^{k}}(z, w)=C_{k}$. But the equation $\Pi_{h^{k}}(z, z)=A_{k}\left(\Pi_{h^{1}}(z, z)\right)^{k}$ is the restriction of a holomorphic equation to the anti-diagonal and therefore extends to all of $M \times \bar{M}$.

3.2. Proof of Theorem 0.4. To prove Theorem 0.4 we need to relate convolution powers of $\mu_{k}^{z}$ and the two conditions in Proposition 0.5.

Define the lattice path 'partition function': For $\alpha \in k P \cap \mathbb{Z}^{m}$,

$$
\mathcal{P}_{k}(\alpha):=\sum_{\left(\beta_{1}, \ldots, \beta_{k}\right): \beta_{j} \in P, \beta_{1}+\cdots+\beta_{k}=\alpha} \prod_{j=1}^{k} \frac{1}{\mathcal{Q}\left(\beta_{j}\right)} .
$$

Then, we have

LEMMA 3.1.

$$
\Pi_{h^{k}}(z, z)=A_{k}\left(\Pi_{h^{1}}(z, z)\right)^{k} \Longleftrightarrow \mathcal{P}_{k} \mathcal{Q}_{k}=A_{k} .
$$

Proof. Recall from (19) that

$$
F_{h^{k}}(z, w)=\sum_{\alpha \in k P \cap \mathbb{Z}^{m}} \frac{z^{\alpha} \bar{w}^{\alpha}}{Q_{h^{k}}(\alpha)}
$$


where $Q_{h^{k}}(\alpha)$ is defined in (9), and that

$$
\Pi_{h^{k}}(x, y)=F_{h^{k}}(z, w) e_{L}^{k}(z) \overline{e_{L}^{k}(w)} .
$$

On the other hand, by definition of the partition function, we also have

$$
F_{h^{1}}^{k}(x, y)=\sum_{\alpha \in k P \cap \mathbb{Z}^{m}} \mathcal{P}_{k}(\alpha) z^{\alpha} \bar{w}^{\alpha}
$$

If we contract the diagonal values of each kernel with the metric, the hypothesis of the Lemma gives,

$$
F_{h^{k}}(z, z)=A_{k}\left(F_{h^{1}}(z, z)\right)^{k}
$$

and comparing coefficients of the monomials completes the proof.

Next, we evaluate the the Fourier transform $\mathcal{F}_{x \rightarrow \xi}\left(\mu_{1}\right)^{* k}$ of the convolution powers of $\mu_{1}^{z}$.

LEMMA 3.2. We have,

$$
\mathcal{F}_{x \rightarrow \xi}\left(\mu_{1}^{z}\right)^{* k}=\frac{1}{\left(\Pi_{h^{1}}(z, z)\right)^{k}} \sum_{\alpha \in k P \cap \mathbb{Z}^{m}} \mathcal{P}_{k}(\alpha) e^{i\langle\alpha, \xi\rangle} .
$$

Proof. By definition of the partition function (54),

$$
\begin{aligned}
\mathcal{F}_{x \rightarrow \xi}\left(\mu_{1}^{z}\right)^{* k}=\left(\mathcal{F} \mu_{1}^{z}\right)^{k}(\xi) & =\left(\sum_{\alpha \in P} \frac{\mathcal{P}_{h^{1}}(\alpha, z)}{\Pi_{h^{1}}(z, z)} e^{i\langle\alpha, \xi\rangle}\right)^{k} \\
& =\frac{1}{\left(\Pi_{h^{1}}(z, z)\right)^{k}} \sum_{\alpha \in k P \cap \mathbb{Z}^{m}} \mathcal{P}_{k}(\alpha) e^{i\langle\alpha, \xi\rangle}
\end{aligned}
$$

The following Lemma is the main step in the proof of Theorem 0.4.

LeMma 3.3. $\mu_{k}^{z}=\left(\mu_{1}^{z}\right)^{* k}$ for all $k$ and all $z \in M^{o}$ if and only if

$$
\frac{\Pi_{h^{k}}(z, z)}{\left(\Pi_{h^{1}}(z, z)\right)^{k}}=A_{k}=\mathcal{P}_{k}(\alpha) \mathcal{Q}_{k}(\alpha), \quad \forall k, \alpha \in k P \cap \mathbb{Z}^{m}, \forall z \in M^{o},
$$

where $A_{k}$ is the constant determined by Proposition 0.5.

Proof. By the definition (2) of $\mathcal{P}_{h^{k}}(\alpha)$,

$$
\begin{aligned}
\mu_{k}^{z}=\left(\mu_{1}^{z}\right)^{* k} & \Longleftrightarrow \sum_{\alpha \in k P \cap \mathbb{Z}^{m}} \frac{\mathcal{P}_{h^{k}}(\alpha, z)}{\Pi_{h^{k}}(z, z)} e^{i\langle\alpha, \xi\rangle}=\left(\sum_{\alpha \in P} \frac{\mathcal{P}_{h^{1}}(\alpha, z)}{\Pi_{h^{1}}(z, z)} e^{i\langle\alpha, \xi\rangle}\right)^{k} \\
& \Longleftrightarrow \quad \frac{\mathcal{P}_{h^{k}}(\alpha, z)}{\Pi_{h^{k}}(z, z)}=\frac{\mathcal{P}_{h^{1}}(\alpha, z)^{k}}{\left(\Pi_{h^{1}}(z, z)\right)^{k}}, \quad \forall \alpha \in k P \cap \mathbb{Z}^{m} \\
& \Longleftrightarrow \quad \frac{1}{\mathcal{Q}_{h^{k}}(\alpha) \Pi_{h^{k}}(z, z)}=\frac{1}{\left(\Pi_{h^{1}}(z, z)\right)^{k}} \mathcal{P}(\alpha), \quad \forall \alpha \in k P \cap \mathbb{Z}^{m} \\
& \Longleftrightarrow \frac{\left(\Pi_{h^{1}}(z, z)\right)^{k}}{\Pi_{h^{k}}(z, z)}=\mathcal{P}(\alpha) \mathcal{Q}_{h^{k}}(\alpha), \quad \forall \alpha \in k P \cap \mathbb{Z}^{m} .
\end{aligned}
$$

Equality is only possible if the left side is independent of $z$ and the right side is independent of $\alpha$. Therefore, both must be a constant, and the left side must be the constant $A_{k}$ determined by Proposition 0.5.

We now complete the proof of Theorem 0.4. First assume that $\mu_{k}^{z}=\left(\mu_{1}^{z}\right)^{* k}$ for all $k$ and all $z \in M^{o}$. Then by Lemma 3.3, $\frac{\Pi_{h^{k}}(z, z)}{\left(\Pi_{h^{1}}(z, z)\right)^{k}}=A_{k}$ for all $k$ and $z \in M^{o}$ ) (hence for $z \in M$ ). Then, by Proposition 0.5, $\Pi_{h^{k}}(z, z)$ is a constant for all $k$, say $D_{k}$. The constant $D_{k}$ is determined by integrating and as in Lemma $0.5, D_{k}=\frac{1}{V o l(M)} \operatorname{dim} H^{0}\left(M, L^{k}\right)$. By the Bergman kernel expansion (18), $\omega$ is a CSC metric.

Conversely suppose that $\operatorname{Hilb}_{\mathrm{k}}(\mathrm{h})$ is balanced for each $k$, i.e. that $\Pi_{h^{k}}(z)$ is constant. By Proposition 0.5, $\frac{\Pi_{h^{k}}(z, z)}{\left(\Pi_{h^{1}}(z, z)\right)^{k}}=A_{k}$ for all $k$ and $z \in M^{o}$ and by Lemma $3.3, \mu_{k}^{z}=\left(\mu_{1}^{z}\right)^{* k}$ for all $k$. 
3.3. Differential entropy of Gaussian measures on $H^{0}\left(M, L^{k}\right)$ : Proof of Proposition 0.7. Proposition 0.7 is of a different nature from the preceding results, since it concerns Gaussian measures on $H^{0}\left(M, L^{k}\right)$ induced by Hermitian metrics on $K$, rather than the toric measures $d \mu_{k}^{z}$. But it is related in that both concern entropies of probability measures induced by Kähler metrics. The proof is rather simple, because we may reduce it to results of Donaldson on balanced metrics.

Proof. The entropy $H\left(\gamma_{P} \mid \gamma_{I}\right)$ of this Gaussian measure relative to that of the background is $-\log \operatorname{det} P$.

In the case of a toric Kähler manifold, we may represent an inner product by the norming constants $Q_{h_{k}}(\alpha)$. In fact the toric Gaussian measure is the product measure

$$
\prod_{\alpha \in k P \cap \mathbb{Z}^{m}} \sqrt{Q_{h_{k}}(\alpha)} e^{-\left\langle Q_{h_{k}}(\alpha)^{-1} x, x\right\rangle} d x
$$

Then $\operatorname{det} P=\prod_{\alpha \in k P \cap \mathbb{Z}^{m}} Q_{h_{k}}(\alpha)$. It follows that the differential entropy of the associated Gaussian measure is

$$
H\left(\gamma_{\vec{Q}_{h_{k}}}\right)=-\log \operatorname{det} \operatorname{Hilb}_{\mathrm{k}}(\mathrm{h})=-\sum_{\alpha \in \mathrm{kP} \cap \mathbb{Z}^{\mathrm{m}}} \log \mathrm{Q}_{\mathrm{h}_{\mathrm{k}}}(\alpha) .
$$

Interestingly, (57) is the functional $\mathcal{L}$ introduced by Donaldson in [D05, (10)]. In [D05, Lemma 2] and [D05, Corollary 1] it is proved that a metric is balanced if and only if it is a critical point of the functional $\widetilde{\mathcal{L}}=\mathcal{L}-\frac{d}{V} I$ on the space $\mathcal{K}$ of Kähler metrics in the fixed $(1,1)$ class. In fact, as explained there, $\delta \mathcal{L}$ vanishes for all $\delta \phi$ of integral zero if and only if the density of states $\Pi_{h^{k}}(z)$ is a constant. The second term $-\frac{d}{V} I$ is only to fix the undetermined constant in the Kähler potential and may be omitted if we work with global potentials on the open orbit.

\section{REFERENCES}

[Ab98] M. Abreu, Kähler geometry of toric varieties and extremal metrics. Internat. J. Math. 9 (1998), no. 6, $641-651$.

[Ab03] M. Abreu, Kähler geometry of toric manifolds in symplectic coordinates. Symplectic and contact topology: interactions and perspectives (Toronto, ON/Montreal, QC, 2001), 1-24, Fields Inst. Commun., 35, Amer. Math. Soc., Providence, RI, 2003.

[CG12] J. Cichon and Z. Golebiewski, On Bernoulli sums and Bernstein polynomials, Broutin, Nicolas and Devroye, Luc. 23rd International Meeting on Probabilistic, Combinatorial, and AsymptoticMethods in the Analysis of Algorithms (AA 12), 2012, Montreal, Canada. Discrete Mathematics and Theoretical Computer Science, DMTCS Proceedings vol. AQ, 23rd Intern. Meeting on Probabilistic, Combinatorial, and Asymptotic Methods for the Analysis of Algorithms (AofA12), pp.179-190, (2012).

[D02] S. K. Donaldson, Scalar curvature and stability of toric varieties. J. Differential Geom. 62 (2002), no. 2, 289-349.

[D05] S. K. Donaldson, Scalar curvature and projective embeddings. II. Q. J. Math. 56 (2005), no. 3, 345-356.

[D08] S. K. Donaldson, Kähler geometry on toric manifolds, and some other manifolds with large symmetry. Handbook of geometric analysis. No. 1, 29-75, Adv. Lect. Math. (ALM), 7, Int. Press, Somerville, MA, 2008.

[DK] M. R. Douglas and S. Klevtsov, Black holes and balanced metrics, arXiv: 0811.0367.

[D96] A. G. Dayachkov, Asymptotics of the Shannon entropy for sums of independent random variables, Fundam. Prikl. Mat., 1996, Volume 2, Issue 4, 10191028

[F12] R. Feng, Szasz analytic functions and non-compact Kähler toric manifolds. J. Geom. Anal. 22 (2012), no. 1, $107-131$.

[Fu W. Fulton, Introduction to Toric Varieties, Annals of Mathematics Studies 131, Princeton Univ. Press, Princeton, NJ, 1993.

[GK] B.V. Gnedenko and A.N. Kolmogorov, Limit distributions for sums of independent random variables. Addison-Wesley Publishing Co., Reading, Mass.-London-Don Mills., Ont. 1968.

[Gu94] V. Guillemin, Kaehler structures on toric varieties. J. Differential Geom. 40 (1994), no. 2, 285-309.

[GS82] V. Guillemin and S. Sternberg, Geometric quantization and multiplicities of group representations. Invent. Math. 67 (1982), no. 3, 515-538.

[JSz99] P. Jacquet and W. Szpankowski, Entropy computations via analytic de-Poissonization. IEEE Trans. Inform. Theory 45 (1999), no. 4, 1072-1081.

[K98] Knessl, C. Integral representations and asymptotic expansions for Shannon and Renyi entropies. Appl. Math. Lett. 11 (1998), no. 2, 69-74.

$[\mathrm{KS}] \quad$ L. B. Koralov and Ya.G. Sinai, Theory of probability and random processes. Second edition. Universitext. Springer, Berlin, 2007.

[M87] T. Mabuchi, Einstein-Kähler forms, Futaki invariants and convex geometry on toric Fano varieties. Osaka J. Math. 24 (1987), no. 4, 705-737.

[Mat78] Matveev, P. (1978). The entropy of the multinomial distribution. Teor. Verojatnost. i Primenen. 23 196-198. 
[SO] Shepp, L. A.; Olkin, I. Entropy of the sum of independent Bernoulli random variables and of the multinomial distribution. Contributions to probability, pp. 201-206, Academic Press, New York-London, 1981.

[STZ03] B. Shiffman, T. Tate, and S. Zelditch, Harmonic analysis on toric varieties. Explorations in complex and Riemannian geometry, 267-286, Contemp. Math., 332, Amer. Math. Soc., Providence, RI, 2003.

[Sh] A.N. Shiryaev, Probability. Third edition Graduate Texts in Mathematics, 95. Springer, New York, 2016.

[SoZ07] J. Song and S. Zelditch, Steve Convergence of Bergman geodesics on CP1. Festival Yves Colin de Verdiere. Ann. Inst. Fourier (Grenoble) 57 (2007), no. 7, 2209-2237.

[SoZ12] J. Song and S. Zelditch, Steve Test configurations, large deviations and geodesic rays on toric varieties. Adv. Math. 229 (2012), no. 4, 2338-2378.

[SoZ10] J. Song and S. Zelditch, Bergman metrics and geodesics in the space of Kähler metrics on toric varieties, Anal. PDE 3 (2010), no. 3, 295-358. (arXiv:0707.3082).

[VD98] P.A. Vilenkin and A.G. Deyachkov, Asymptotics of Shannon and Renyi entropies for sums of independent random variables. (Russian) Problemy Peredachi Informatsii 34 (1998), no. 3, 17-31; translation in Problems Inform. Transmission 34 (1998), no. 3, 219-232.

[W15] F. Wang, A volume stability theorem on toric manifolds with positive Ricci curvature. Proc. Amer. Math. Soc. 143 (2015), no. 8, 3613-3618.

[WZ04] X. J. Wang and X. Zhu, Khler-Ricci solitons on toric manifolds with positive first Chern class. Adv. Math. 188 (2004), no. $1,87-103$.

[W] Differential entropy. Wikipedia article.

[Z09] S. Zelditch, Bernstein polynomials, Bergman kernels and toric Kaehler varieties, J. Symplectic Geom. 7 (2) (2009) 51-76.

[ZZ18] S. Zelditch and P.Zhou, Central Limit theorem for toric Kähler manifolds, to appear in the PAMQ volume in honor of D.H. Phong (arXiv:1802.08501).

[ZZ19] S. Zelditch and P. Zhou, Interface asymptotics of partial Bergman kernels on S1-symmetric Khler manifolds. J. Symplectic Geom. 17 (2019), no. 3, 793-856.

Department of Mathematics, Northwestern University, Evanston, IL 60208, USA

Email address: zelditch@math.northwestern.edu 